\title{
Spatially resolved origin of millimeter-wave linear polarization in the nuclear region of $3 \mathrm{C} 84^{\star}$
}

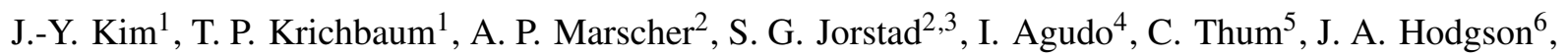
N. R. MacDonald ${ }^{1}$, E. Ros ${ }^{1}$, R.-S. Lu ${ }^{1,7}$, M. Bremer ${ }^{8}$, P. de Vicente ${ }^{9}$, M. Lindqvist ${ }^{10}$, S. Trippe ${ }^{11}$, and J. A. Zensus ${ }^{1}$

${ }^{1}$ Max-Planck Institut für Radioastronomie, Auf dem Hügel 69, 53121 Bonn, Germany e-mail: jykim@mpifr-bonn.mpg.de

2 Institute for Astrophysical Research, Boston University, 725 Commonwealth Avenue, Boston, MA 022, USA

3 Astronomical Institute, St. Petersburg State University, Universitetskij Pr. 28, Petrodvorets, 198504 St. Petersburg, Russia

4 Instituto de Astrofísica de Andalucía (CSIC), Apartado 3004, 18080 Granada, Spain

5 Instituto de Radio Astronomía Millimétrica, Avenida Divina Pastora, 7, Local 20, 18012 Granada, Spain

${ }^{6}$ Korea Astronomy and Space Science Institute, 776 Daedeokdae-ro, Yuseong-gu, Daejeon 30455, Korea

7 Shanghai Astronomical Observatory, Chinese Academy of Sciences, 80 Nandan Road, Shanghai 200030, PR China

8 Institut de Radio Astronomie Millimétrique, 300 rue de la Piscine, Domaine Universitaire, 38406 Saint-Martin-d'Hères, France

9 Observatorio de Yebes (IGN), Apartado 148, 19180 Yebes, Spain

${ }_{10}$ Department of Space, Earth and Environment, Chalmers University of Technology, Onsala Space Observatory, 43992 Onsala, Sweden

11 Department of Physics and Astronomy, Seoul National University, 1 Gwanak-ro, Gwanak-gu, Seoul 08826, Korea

Received 28 February 2018 / Accepted 16 November 2018

\begin{abstract}
We report results from a deep polarization imaging of the nearby radio galaxy $3 \mathrm{C} 84$ (NGC 1275). The source was observed with the Global Millimeter VLBI Array (GMVA) at $86 \mathrm{GHz}$ at an ultrahigh angular resolution of $50 \mu$ as (corresponding to $\sim 200 R_{\mathrm{s}}$ ). We also add complementary multiwavelength data from the Very Long Baseline Array (VLBA; 15 and $43 \mathrm{GHz}$ ) and from the Atacama Large Millimeter/submillimeter Array (ALMA; 97.5, 233.0 and 343.5 GHz). At 86 GHz, we measured a fractional linear polarization of $\sim 2 \%$ in the VLBI core region. The polarization morphology suggests that the emission is associated with an underlying limbbrightened jet. The fractional linear polarization is lower at 43 and $15 \mathrm{GHz}(\sim 0.3-0.7 \%$ and $<0.1 \%$, respectively). This suggests an increasing linear polarization degree toward shorter wavelengths on VLBI scales. We also obtain a large rotation measure (RM) of $\sim 10^{5-6} \mathrm{rad} \mathrm{m}^{2}$ in the core at $\gtrsim 43 \mathrm{GHz}$. Moreover, the VLBA $43 \mathrm{GHz}$ observations show a variable RM in the VLBI core region during a small flare in 2015. Faraday depolarization and Faraday conversion in an inhomogeneous and mildly relativistic plasma could explain the observed linear polarization characteristics and the previously measured frequency dependence of the circular polarization. Our Faraday depolarization modeling suggests that the RM most likely originates from an external screen with a highly uniform RM distribution. To explain the large RM value, the uniform RM distribution and the RM variability, we suggest that the Faraday rotation is caused by a boundary layer in a transversely stratified jet. Based on the RM and the synchrotron spectrum of the core, we provide an estimate for the magnetic field strength and the electron density of the jet plasma.
\end{abstract}

Key words. galaxies: active - galaxies: jets - galaxies: individual: NGC 1275 - galaxies: individual: 3 C 84 techniques: interferometric - techniques: polarimetric

\section{Introduction}

Magnetic fields in the vicinity of central black holes (BHs) could play a significant role in the formation, collimation and acceleration of relativistic jets in active galactic nuclei (AGNs; e.g., Blandford \& Znajek 1977; Blandford \& Payne 1982). Polarization-sensitive very-long-baseline interferometry (VLBI) is an important technique to study the topology and strength of the magnetic fields at the base of AGN jets. Synchrotron theory predicts linear polarization of up to $m_{\mathrm{L}} \sim 70 \%$ for optically thin emission and well ordered magnetic fields (Pacholczyk 1970). However, VLBI observations typically find

* The reduced images (FITS format) are only available at the CDS via anonymous ftp to cdsarc.u-strasbg.fr $(130.79 .128 .5)$ or via http://cdsarc.u-strasbg.fr/viz-bin/qcat?J/A+A/622/ A196. much lower linear polarizations $\left(m_{\mathrm{L}} \lesssim 5 \%\right.$ ) in the VLBI cores of AGN jets (Lister \& Homan 2005; Jorstad et al. 2007). The origin of the weak linear polarization may be due to (i) high synchrotron self-absorption opacity, (ii) a large Faraday depth (e.g., Taylor et al. 2006) and (iii) highly disordered magnetic fields within the observing beam, for example induced by a turbulent plasma (e.g., Marscher 2014). The observed linear polarization can also be reduced by bandwidth depolarization if Faraday rotation is large. Observations at millimeter wavelengths help to overcome the opacity barrier and are less affected by Faraday depolarization than in the centimeter $(\mathrm{cm})$ bands. Furthermore, owing to their small beam size, VLBI observations at millimeter wavelengths (mm-VLBI) have a small in-beam depolarization, in comparison to single-dish or connected interferometer array observations. Thus, mm-VLBI polarimetry is a suitable technique to observe and study the polarization properties of compact radio sources (see also Boccardi et al. 2017). 
Table 1. Summary of the polarimetric VLBI observations of 3C 84 in May 2015.

\begin{tabular}{|c|c|c|c|c|c|c|c|c|c|}
\hline $\begin{array}{c}\text { Stations } \\
\text { (1) }\end{array}$ & $\begin{array}{c}\text { Epoch } \\
(2) \\
{[\mathrm{yy} / \mathrm{mm} / \mathrm{dd}]}\end{array}$ & $\begin{array}{c}v_{\mathrm{obs}} \\
(3) \\
{[\mathrm{GHz}]}\end{array}$ & $\begin{array}{c}\text { Beam } \\
(4) \\
{[\mathrm{mas}, \mathrm{deg}]}\end{array}$ & $\begin{array}{c}I_{\text {peak }} \\
(5) \\
{\left[\mathrm{Jy} \mathrm{bm}^{-1}\right]}\end{array}$ & $\begin{array}{c}S_{\text {Ptot }} \\
(6) \\
{[\mathrm{Jy}]} \\
\end{array}$ & $\begin{array}{c}\sigma_{\mathrm{I}} \\
(7) \\
{\left[\mathrm{mJy} \mathrm{bm}^{-1}\right]}\end{array}$ & $\begin{array}{c}P I_{\text {peak }} \\
(8) \\
{\left[\mathrm{mJy} \mathrm{bm}^{-1}\right]}\end{array}$ & $\begin{array}{c}P_{\text {tot }} \\
(9) \\
{[\mathrm{mJy}]}\end{array}$ & $\begin{array}{c}\sigma_{P} \\
(10) \\
{\left[\mathrm{mJy} \mathrm{bm}^{-1}\right]}\end{array}$ \\
\hline \multicolumn{10}{|l|}{ VLBA(8) } \\
\hline$+\mathrm{GBT}+\mathrm{EB}+\mathrm{ON}$ & $2015 / 05 / 16$ & 86.252 & $0.048 \times 0.14(-16.2)$ & 1.82 & 12.0 & 0.53 & 27.7 & 42.0 & 5.03 \\
\hline $\operatorname{VLBA}(10)$ & $2015 / 05 / 11$ & 43.115 & $0.15 \times 0.30(1.02)$ & 3.82 & 17.0 & 1.24 & 12.3 & 16.8 & 1.75 \\
\hline $\operatorname{VLBA}(10)$ & $2015 / 05 / 18$ & 15.352 & $0.42 \times 0.63(-8.74)$ & 4.00 & 28.8 & 1.30 & $<2.64$ & - & 0.88 \\
\hline
\end{tabular}

Notes. (1) Antennas participating in each epoch. Numbers in parentheses indicate the number of participating VLBA antennas. (2) The observing epoch in year/month/day format. (3) Center frequency. (4) The restoring beam for the uniform weighting. Minor axis $\times$ major axis in mas (the position angle in degrees measured counterclockwise from north). (5) Total intensity peak (in units of Jy per beam). (6) Total flux density integrated over the entire VLBI image. (7) Total intensity rms level. (8) Linear polarization intensity peak (upper limit for $3 \sigma$ significance). (9) Integrated linearly polarized flux density of the core region obtained by the quadratic sum of the integrated Stokes $Q$ and $U$ flux densities in the region where significant linear polarization intensities are found ("-" means no significant linear polarization in the region displayed in Figs. 2 and 3). (10) The polarization intensity noise level obtained as described in Sect. 2.1.3.

The radio galaxy $3 \mathrm{C} 84$ (Perseus A, NGC 1275) is an intriguing source whose linear polarization in the core region at $\mathrm{cm}$ wavelengths is extremely weak $(<0.1 \%$ at $\leq 15 \mathrm{GHz}$; Taylor et al. 2006). This is much lower than typically observed in other AGN jets (Lister \& Homan 2005). The source is also relatively nearby, at a redshift of $z=0.0176$ and a luminosity distance of $d_{\mathrm{L}}=75 \mathrm{Mpc}$ (Strauss et al. 1992) ${ }^{1}$. The mass of its central supermassive black hole (SMBH) has been estimated to be $M_{\mathrm{BH}} \sim 9 \times 10^{8} M_{\odot}$ (Scharwächter et al. 2013). The combination of proximity and large black hole mass makes 3C 84 an ideal candidate to study the jet base and its polarization properties with the highest possible angular and spatial resolution, using global mm-VLBI. For the adopted distance an angular scale of $50 \mu$ as corresponds to a linear scale of $\sim 0.0175 \mathrm{pc}$ or $\sim 203$ Schwarzschild radii $\left(R_{\mathrm{s}}\right)$.

At 1.3 and $0.9 \mathrm{~mm}$ wavelengths (230 and $341 \mathrm{GHz})$, Plambeck et al. (2014) report a typical degree of linear polarization $m_{\mathrm{L}} \approx 1 \%$ using the Combined Array for Research in Millimeter Astronomy (CARMA) and the Submillimeter Array (SMA) with arcsecond scale angular resolution. The authors also find a large rotation measure (RM) of $\sim 9 \times 10^{5} \mathrm{rad} \mathrm{m}^{-2}$. IRAM $30 \mathrm{~m}$ Telescope observations at 86 and $230 \mathrm{GHz}$ reveal a typical RM $\sim 7 \times 10^{4} \mathrm{rad} \mathrm{m}^{-2}$ (Agudo et al. 2014, 2018b). Both Plambeck et al. (2014) and Agudo et al. (2018b) report polarization variability on monthly timescales at $\mathrm{mm}$ wavelengths, implying a small emission region (apparent size $\lesssim 1 c \times 1$ month $0.025 \mathrm{pc} \sim 0.1 \mathrm{mas})$.

At $\mathrm{cm}$ wavelengths and on milliarcsecond scales, however, only low-level polarization is observed. As mentioned above, Very Long Baseline Array (VLBA) observations of the VLBI core of 3C 84 reveal a linear polarization of $m_{\mathrm{L}} \lesssim 0.1 \%$ at $15 \mathrm{GHz}$ (upper limit) and $m_{\mathrm{L}} \sim 0.2 \%$ at $22 \mathrm{GHz}$ (Taylor et al. 2006). It was suggested that Faraday depolarization with $\mathrm{RM} \gtrsim$ $10^{4} \mathrm{rad} \mathrm{m}^{-2}$ could be an explanation (Taylor et al. 2006). The high RM is also consistent with the non-VLBI measurements (Plambeck et al. 2014; Agudo et al. 2018b). However, the limited angular resolution of the previous observations does not yet allow us to precisely localize the polarized emission region nor unambiguously determine the physical origin of the high RM. Observations with the Global Millimeter-VLBI Array (GMVA) help to overcome this limitation. The observing frequency of $86 \mathrm{GHz}$ nicely bridges the gap between the 5 and $22 \mathrm{GHz}$ and 1 We assume $\Lambda$ CDM cosmology with $H_{0}=71 \mathrm{~km} \mathrm{~s}^{-1} \mathrm{Mpc}^{-1}, \Omega_{\Lambda}=$
0.73 and $\Omega_{\mathrm{M}}=0.27$.
$230 \mathrm{GHz}$ observations. This will help to connect the results from the previous studies (e.g., Aller et al. 2003; Agudo et al. 2010, 2018a; Trippe et al. 2012; Plambeck et al. 2014; Nagai et al. 2017), in order to form a self-consistent physical jet polarization model from the $\mathrm{cm}$ to the $\mathrm{mm}$ regime.

In this paper, we present a new study of the polarization properties of 3C 84 based on GMVA observations at $86 \mathrm{GHz}$ and supplemented by close-in-time VLBA observations at $43 \mathrm{GHz}$ and $15 \mathrm{GHz}$. In Sect. 2 we describe details of the VLBI observations and the data reduction. Our main findings from the observations are summarized in Sect. 3. The physical implications are discussed in Sect. 4. In Sect. 5 we summarize the results.

\section{Observations and data reduction}

\subsection{GMVA $86 \mathrm{GHz}$ data}

The source 3C 84 was observed with the GMVA at $86 \mathrm{GHz}$ in dual circular polarization in May 2015. The source was observed as one of the $\gamma$-ray emitting AGN sources, which are monitored by semiannual GMVA observations that complement the VLBABU-BLAZAR monitoring program (Jorstad et al. 2017; for the GMVA monitoring see e.g., Rani et al. 2015; Hodgson et al. 2017; Casadio et al. 2017). In this GMVA campaign a total of 11 antennas observed 3C 84: Brewster (BR), Effelsberg (EB), Fort Davis (FD), the Green Bank Telescope (GB), Kitt Peak (KP), Los Alamos (LA), Mauna Kea (MK), North Liberty (NL), Onsala (ON), Owens Valley (OV) and Pie Town (PT). The galaxy was observed in full track mode for $8 \mathrm{~h}$. The data were recorded at an aggregate bitrate of $2 \mathrm{Gbps}$ with two-bit digitization. Using the polyphase filterbank, the data were channelized in eight intermediate frequencies (IFs) per polarization, with an IF bandwidth of $32 \mathrm{MHz}$, yielding a total recording bandwidth of $2 \times 256 \mathrm{MHz}$. The data were correlated using the DiFX correlator (Deller et al. 2011) at the Max-Planck-Institut für Radioastronomie (MPIfR) in Bonn, Germany. A summary of details of the observations is given in Table 1 .

\subsubsection{Total intensity calibration and imaging}

The postcorrelation analysis and calibration of the correlated data were done using standard VLBI fringe-fitting and calibration procedures in AIPS (Greisen 1990; see also Martí-Vidal et al. 2012 for the analysis of GMVA data). In the first step of the phase calibration, delay and phase offsets 
between the IFs were removed using high signal-to-noise ratio (S/N) fringe detection (so called manual phase calibration). After the phase alignment across the IFs, the global fringe fitting was performed over the full bandwidth, in order to solve for the delays and rates combining all IFs. We set the S/N threshold to five. The number of detections was maximized when we used a relatively long fringe solution interval of four minutes, which was moved forward in time in one-minute steps. In the next step of the process, we performed the a priori amplitude calibration using the AIPS task APCAL, using a priori calibration information provided by each station (system temperatures $T_{\text {sys }}$ and elevation dependent gain curves). An opacity correction was performed for stations that did not apply this correction in the delivered system temperature values. Finally, the cross-hand phase and delay offsets of the reference station were determined and removed using AIPS task RLDLY. The UVFITS data were then exported outside AIPS using the AIPS task SPLIT by frequencyaveraging the cross power spectra.

At $86 \mathrm{GHz}$, the atmosphere limits the phase coherence. In order to estimate the effect of coherence losses, we fringe-fitted the data once again using much shorter fringe solution intervals comparable to the coherence time. For this purpose, we set the fringe solution interval to $10 \mathrm{~s}$ and adopted the same $\mathrm{S} / \mathrm{N}$ threshold of five. We also used more narrow fringe search windows for delay and rate $( \pm 10 \mathrm{~ns}$ and $\pm 25 \mathrm{mHz}$, respectively). The number of detected scans was reduced dramatically due to the short solution interval. For the detected scans, we found that the short ten-second solution interval did not increase the baseline amplitudes significantly, as long as the phases were not averaged over a longer time interval. (e.g., $\lesssim 10 \%$ of decoherence amplitude loss with ten-second time-averaging).

The frequency-averaged (but not yet time-averaged) data were read into the DIFMAP package (Shepherd et al. 1994) for imaging. We flagged outlying visibilities which were caused by known or previously unrecognized antenna problems (such as pointing and focus errors, and bad weather). After an initial phase self-calibration using a point-source model, the visibility data were coherently time averaged over $10 \mathrm{~s}$. We then imaged the source structure in Stokes $I$ by applying the standard, iterative CLEAN and self-calibration steps (for both phase and amplitude). With careful CLEAN and self-calibrations, our CLEAN model was able to reach the maximum correlated flusx density on the shortest uv-spacings (e.g., $\$ 200 M \lambda$ ). We however note that the limited accuracy of the a-priori calibration for the stations that form the shortest baselines (e.g., PT and LA) could have an effect on the maximum absolute flux density.

In order to correct for a systematic flux density scaling, we combined quasi-simultaneous data from VLBA observations of the source at 15 and $43 \mathrm{GHz}$ (Sect. 2.2) and computed the synchrotron spectrum of the extended jet in the source (at $\sim 2-4$ mas from the VLBI core). We then assumed that the observed spectral index of the extended jet components between 15 and $43 \mathrm{GHz}$ is constant (no spectral break) and thus is the same as at the higher frequencies (i.e., between 43 and $86 \mathrm{GHz}$ ). Based on this assumption and the observed spectral index of $\alpha_{15-43 \mathrm{GHz}} \sim-0.5$ $\left(S \propto v^{+\alpha}\right)$, we estimated a correction factor for the absolute flux density of the source and further calibrated the data. We also checked the accuracy of the absolute flux density by making use of close-in-time single dish observations and connected interferometer flux measurements of the source between 15 and $343.5 \mathrm{GHz}$. From this we concluded that the absolute flux density of the source in this epoch could be uncertain by $\sim 30 \%$ (see Appendix A for details).
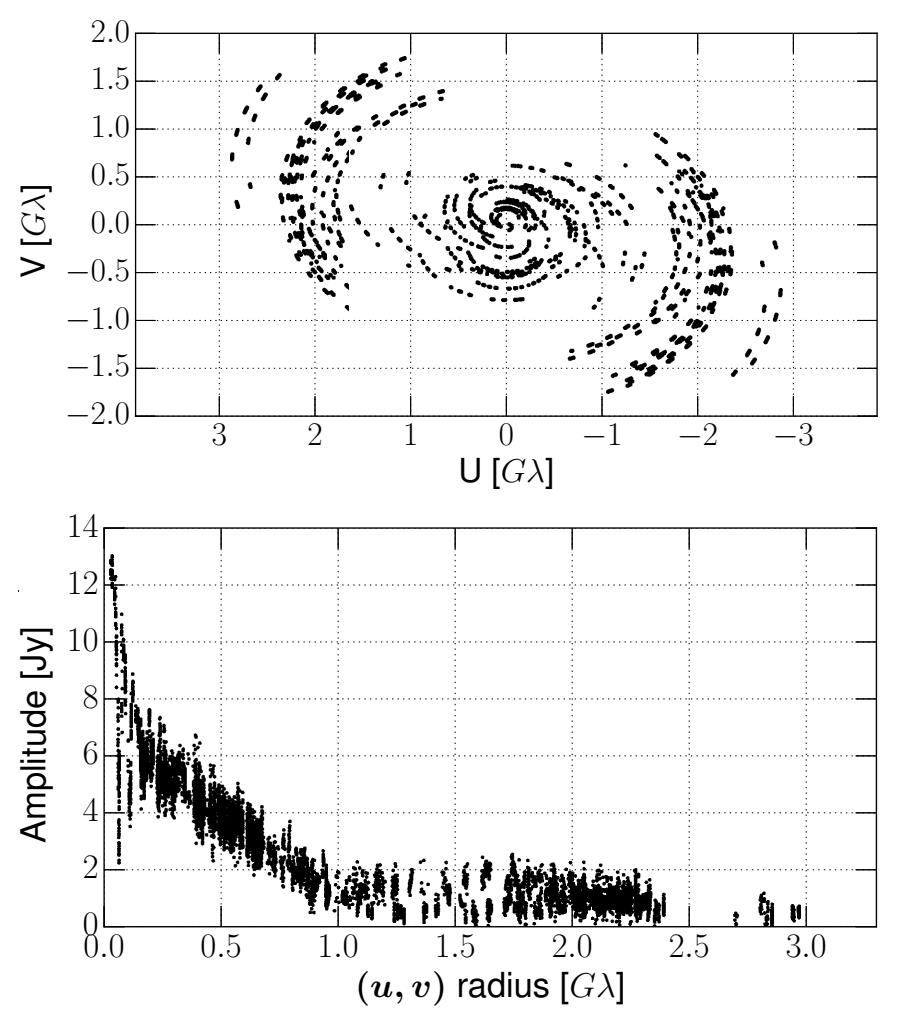

Fig. 1. Stokes $I$ visibilities of the GMVA data. Top panel: $(u, v)$-coverage after the a priori calibration and additional flagging for the imaging. Bottom panel: radial distribution of the visibility amplitudes after the last round of CLEAN and self-calibration. The visibility data were binned at $30 \mathrm{~s}$ for clarity.

We examined the reliability of the CLEAN model and the self-calibration solutions by comparing the closure phases and amplitudes of the a-priori calibrated data and the CLEAN model. In particular, we compared the differences (and ratios) of the closure phases (and closure amplitudes) between the data and the model. The residual closure phases and the closure amplitude ratios were computed for the histograms of the entire data set. We found that the closure quantities of the a priori calibrated data and the CLEAN model agreed within $\sim 10^{\circ}$ for the phases and $\sim 25 \%$ for the amplitude, respectively.

A summary of the characteristics of the final $86 \mathrm{GHz}$ Stokes $I$ image is given in Table 1 . In Fig. 1 we show the $(u, v)$ coverage of the data set and the visibility amplitudes versus the $(u, v)$ distances after all the calibrations.

\subsubsection{Polarization calibration and imaging}

After the total intensity imaging, additional calibration steps were performed in AIPS in order to remove residual gain offsets between the RCP and LCP receivers and the polarization leakage effects. In particular, the antenna instrumental polarization solutions (D-terms) were determined using the AIPs task LPCAL (Leppanen et al. 1995). We derived the D-terms from other more compact calibrator sources including 3C 454.3, BL Lac, CTA 102 and OJ 287, which were observed in the same session. We also derived the D-terms from 3C 84 itself, which is facilitated by (i) its core-dominace at millimeter wavelengths (see Sect. 3) and (ii) wide parallactic angle coverage in this data set. We show two linear polarization images of 3C 84 in Appendix B for D-term solutions derived from CTA 102 and OJ 287. The 
Table 2. Summary of the antenna D-terms at $86 \mathrm{GHz}$.

\begin{tabular}{|c|c|c|c|c|}
\hline \multirow{2}{*}{$\begin{array}{c}\text { Station } \\
(1) \\
\end{array}$} & \multicolumn{2}{|c|}{$\mathrm{RCP}$} & \multicolumn{2}{|c|}{ LCP } \\
\hline & $\begin{array}{c}m \\
{[\%]} \\
(2) \\
\end{array}$ & $\begin{array}{c}\chi \\
\text { [deg] } \\
(3)\end{array}$ & $\begin{array}{c}m \\
{[\%]} \\
(4) \\
\end{array}$ & $\begin{array}{c}\chi \\
\text { [deg] } \\
(5)\end{array}$ \\
\hline BR & $5.8 \pm 2.7$ & $-(106 \pm 16)$ & $7.2 \pm 2.7$ & $-(48 \pm 20)$ \\
\hline EB & $4.5 \pm 2.1$ & $56 \pm 24$ & $2.3 \pm 2.5^{a}$ & $98 \pm 40^{a}$ \\
\hline FD & $7.9 \pm 2.9$ & $23 \pm 13$ & $7.0 \pm 3.6$ & $-(141 \pm 25)$ \\
\hline GB & $1.7 \pm 2.4^{a}$ & $-(162 \pm 46)^{a}$ & $2.6 \pm 1.3$ & $-(66 \pm 44)^{a}$ \\
\hline $\mathrm{KP}$ & $3.1 \pm 2.8$ & $178 \pm 30$ & $3.8 \pm 1.7$ & $88 \pm 14$ \\
\hline LA & $10.7 \pm 1.6$ & $139 \pm 9$ & $10.1 \pm 2.1$ & $32 \pm 13$ \\
\hline MK & $3.9 \pm 3.1$ & $11 \pm 25$ & $4.3 \pm 1.9$ & $-(90 \pm 23)$ \\
\hline NL & $4.8 \pm 1.6$ & $-(167 \pm 21)$ & $3.7 \pm 1.3$ & $70 \pm 13$ \\
\hline ON & $5.9 \pm 1.9$ & $-(178 \pm 27)$ & $4.8 \pm 2.4$ & $-(4 \pm 36)$ \\
\hline OV & $3.6 \pm 2.4$ & $-(48 \pm 38)$ & $5.4 \pm 1.7$ & $-(127 \pm 21)$ \\
\hline PT & $8.2 \pm 5.6$ & $7 \pm 8$ & $9.6 \pm 3.4$ & $-(134 \pm 21)$ \\
\hline
\end{tabular}

Notes. The Green Bank Telescope was used as the reference antenna. The columns show (1) the station code, $(2,3)$ the amplitude $(m)$ and phase $(\chi)$ of the D-terms for the RCP and $(4,5)$ the same for the LCP. ${ }^{(a)}$ Small D-term amplitudes induce large uncertainties in the D-term phases.

maps show a broadly consistent appearance of the polarization. In order to obtain a final set of D-terms, we vector-averaged the D-terms and created the final polarization image of $3 \mathrm{C} 84$ for further analysis. Confidence in the final D-terms was obtained by imaging the polarized structure of the calibrators. Their GMVA polarization images showed good agreement with contemporaneous VLBA $43 \mathrm{GHz}$ polarization images available from the VLBA-BU-BLAZAR database ${ }^{2}$, but with more fine-scale structure seen in the GMVA images. Those images will be published later elsewhere.

The final D-term solutions are shown in Table 2. Their errors are estimated based on the standard deviation of the different $D$ term values for each station and polarization.

The absolute electric vector position angle (EVPA) of 3C 84 was calibrated using OJ 287, 3C 454.3 and BLLac. These calibrators were also observed as part of the POLAMI (Polarimetric Monitoring of AGNs at Millimeter Wavelengths) program $^{3}$ (Agudo et al. 2018a) by the IRAM 30 m Telescope, which was equipped with the XPOL polarimeter (Thum et al. 2008). We estimated the overall uncertainty of the EVPA measurements in GMVA data using the following procedure: first, the measurement error of the $86 \mathrm{GHz}$ EVPA in the IRAM $30 \mathrm{~m}$ Telescope data is $\sim 2-7 \mathrm{deg}$ for different sources and for this particular epoch (see also Agudo et al. 2018a). By deriving the VLBI EVPA correction values from multiple calibrators, we adopted a conservative absolute EVPA uncertainty of $\sim 10 \mathrm{deg}$. Second, the polarization angle of the source and the D-term phases are correlated and uncertainties of the latter could affect the accuracy of the former (see, e.g., Eqs. (3) and (13) of Leppanen et al. 1995). The average phase errors of the D-terms was $\sim 24$ deg (Table 2). Accordingly, we estimated that the corresponding EVPA uncertainty would be $\sim 24 / 2=12 \mathrm{deg}$. Third, we computed the peakto-noise of the polarization features in Stokes $Q$ and $U$ and propagated the errors to estimate the EVPA uncertainty due to the thermal noise. Under the assumption that these three measurement errors are uncorrelated, we can add them in

\footnotetext{
2 https://www.bu.edu/blazars/VLBAproject.html

http://polami.iaa.es/
}

quadrature. For a $\mathrm{S} / \mathrm{N}$ of $\sim 5$, this led to an overall EVPA uncertainty of $\sim 17 \mathrm{deg}$ in this GMVA observation.

\subsubsection{Estimating noise levels in the polarization image}

The Rice bias correction for polarization measurements (see, e.g., Wardle \& Kronberg 1974; Montier et al. 2015a,b) requires an accurate estimation of the $\mathrm{S} / \mathrm{N}$. We simulated the probability density distribution of the noise in the linear polarization intensity image, $\sigma_{\mathrm{P}}$, by performing a Monte-Carlo simulation. First, we computed the $1 \sigma$ noise levels for Stokes $Q$ and $U$ by fitting Gaussian functions to pixel value histograms of sourcefree regions in the Stokes $Q$ and $U$ images. We then simulated Gaussian-distributed noises for $Q$ and $U$ and added them in quadrature to obtain the probability distribution of the polarization noise. By calculating the $68 \%, 97 \%$ and the $99.7 \%$ confidence levels, we obtained the noise values of 5.7, 9.5 and $14.0 \mathrm{mJy} \mathrm{beam}^{-1}$ for the conventional $1 \sigma, 2 \sigma$, and $3 \sigma$ levels, respectively. Based on this, we adopted $1 \sigma_{\mathrm{P}} \sim 5 \mathrm{mJy} \mathrm{beam}^{-1}$ at $86 \mathrm{GHz}$.

We note that this value is higher than the noise in the Stokes $I$ image $\left(\sigma_{\mathrm{I}} \sim 0.5 \mathrm{mJy}\right.$ beam $\left.^{-1}\right)$. In the ideal case, the noise level of a linear polarization map, $\sigma_{\mathrm{P}}$, is expected to be comparable to or lower than that of the total intensity map, $\sigma_{\mathrm{I}}$, because the latter is often dynamic-range limited (see, e.g., Thompson et al. 2017). However, this is not the case if systematic polarization calibration errors dominate the polarization noise (e.g., due to errors in the D-terms). Thus, we checked if the increased noise level in the linear polarization map could be due to uncertain D-term solutions. Following Roberts et al. (1994; see also Hovatta et al. 2012), we estimated the error from D-term calibration as follows:

$\sigma_{\text {Dterm }}=\frac{\Delta m}{\sqrt{N_{\text {ant }} \times N_{\mathrm{IF}} \times N_{\text {scan }}}} \sqrt{I^{2}+\left(0.3 \times I_{\text {peak }}\right)^{2}}$,

where $\Delta m$ is the error of the D-term amplitudes, $N_{\text {ant }}$ is the number of the antennas, $N_{\mathrm{IF}}$ is the number of IFs, $N_{\text {scan }}$ is the total number of independent scans with different parallactic angles, $I$ is the total intensity pixel values and $I_{\text {peak }}$ is the peak value of the total intensity image. As shown in Table 2, the D-term amplitudes are uncertain by $\sim 2-4 \%$ (the largest uncertainty of $\sim 5 \%$ is found for RCP at PT). Therefore, we assumed a characteristic value of $\Delta m \approx 0.03$. For the GMVA data of 3C 84, we adopt $N_{\text {ant }}=11$ and $N_{\mathrm{IF}}=8$. The number of independent scans including all the antennas was $N_{\text {scan }}=8$. This gave us a characteristic $\sigma_{\text {Dterm }} \sim 10^{-3} \times I_{\text {peak }}$. For $I_{\text {peak }}=1.8 \mathrm{Jy} \mathrm{beam}^{-1}$, we obtain $\sigma_{\text {Dterm }} \sim 2 \mathrm{mJy}_{\text {beam }}{ }^{-1}$. The order of $\sigma_{\text {Dterm }}$ appears to be in agreement with our estimation of $\sigma_{\mathrm{P}}$, suggesting that residual polarization leakages may dominate the polarization noise in our $86 \mathrm{GHz}$ image.

\subsection{Contemporaneous 43- and $15 \mathrm{GHz}$ VLBA data}

On short timescales of approximately one week or less, the structural variability of 3C 84 is likely negligible because of the known slow apparent jet speed of $0.1 c \sim 0.1$ mas yr $=$ $2 \mu \mathrm{as}$ week $^{-1}$ in the core region of 3C 84 (Walker et al. 1994; Suzuki et al. 2012). This allows us to combine the $86 \mathrm{GHz}$ data with quasi-contemporaneous observations at other frequencies.

We made use of archival polarimetric VLBA data of 3C 84 provided by the MOJAVE program ${ }^{4}(15 \mathrm{GHz}$; Lister \& Homan 2005) and the VLBA-BU-BLAZAR monitoring program

\footnotetext{
4 https://WwW.physics.purdue. edu/MOJAVE/
} 
(43 GHz; Jorstad et al. 2017). We have chosen a set of 15- and $43 \mathrm{GHz}$ data which were obtained closest in time to the GMVA observations. The archival data were gathered with the same dual polarization setup (RCP and LCP) and the same total bandwidth of $2 \times 256 \mathrm{MHz}$. The Stokes $I, Q$ and $U$ maps again were obtained using DIFMAP, averaging the visibilities over the whole observing bandwidth. At these two frequencies, we estimated the polarization image noise levels $\sigma_{\mathrm{P}}$ in the same way as described in Sect. 2.1.3. The $\sigma_{\mathrm{P}}$ values were comparable to $\sigma_{\mathrm{I}}$, indicating that at these lower frequencies the systematic uncertainties in the linear polarization due to errors in the D-terms were small. A summary of the two data sets is given in Table 1 .

For the absolute EVPA measurement error (i.e., those arising from the D-term calibration and absolute EVPA correction), we adopted $5 \mathrm{deg}$ and $10 \mathrm{deg}$ at 15 and $43 \mathrm{GHz}$ based on Lister \& Homan (2005) and Jorstad et al. (2005), respectively. The overall uncertainty for the EVPA was then estimated in the same way as we described in Sect. 2.1.2 by adding the systematic and thermal errors in quadrature.

In addition, the VLBA-BU-BLAZAR program provides the VLBA $43 \mathrm{GHz}$ data at four different frequencies in the $43 \mathrm{GHz}$ band $(43.0075,43.0875,43.1515$ and $43.2155 \mathrm{GHz})$. Their polarization calibration was performed separately for each IF with respect to the D-terms and EVPA values (see Sect. 3.1 of Jorstad et al. 2005). Therefore, we created additional $43 \mathrm{GHz}$ polarization images of the source for each of the four IFs separately, in order to resolve the $n \pi$ angle ambiguity in the rotation measure analysis and to check for a possible large EVPA rotation inside the $43 \mathrm{GHz}$ band.

\subsection{Noncontemporaneous VLBA data at $43 \mathrm{GHz}$}

We also analyzed other VLBA $43 \mathrm{GHz}$ data of $3 \mathrm{C} 84$ available from the BU database in order to study the time variability of the total flux and the linear polarization over a longer period. We specifically focused on observations performed in 2015 (eight epochs; Feb., Apr., May, Jun., Jul., Aug., Sep. and Dec.). We imaged the source again by applying some additional data flagging when systematically large errors were clearly seen in some antennas or scans. The full-polarization images available from the $\mathrm{BU}$ database reveal a weak polarization feature in the core region of 3C 84 in all eight epochs in 2015. In our imaging, we recovered a very similar polarization morphology in most epochs, however some epochs (Feb., Jun., Aug. and Dec.) appeared to be more limited in polarization fidelity, possibly due to the limited number of scans or other systematic errors. Therefore, we did not include these polarization images in our analysis. Overall, repeatedly appearing and therefore reliable polarization features have been detected in four (Apr., May, Jul. and Sep.) out of the eight epochs. We also measured the unpolarized core flux for all eight epochs using the MODELFIT routine implemented in DIFMAP and analyzed their uncertainties following Schinzel et al. (2012; see Sect. 2.5). We summarize details for these data in Appendix C.

\subsection{Contemporaneous ALMA data}

Finally, we made use of contemporaneous (May 2015) linear polarization measurements of 3C 84 from the Atacama Large Millimeter/submillimeter Array (ALMA) Calibrator Source Catalogue $^{5}$. The source was observed at $97.5,233.0$ and $343.5 \mathrm{GHz}$

\footnotetext{
https://almascience.eso.org/alma-data/ calibrator-catalogue
}

Table 3. Summary of the ALMA archival data.

\begin{tabular}{|c|c|c|c|c|}
\hline $\begin{array}{c}\text { Epoch } \\
(1) \\
{[\text { yyyy/mm/dd] }}\end{array}$ & $\begin{array}{c}v_{\mathrm{obs}} \\
(2) \\
{[\mathrm{GHz}]}\end{array}$ & $\begin{array}{l}S_{\text {tot }} \\
(3) \\
{[\mathrm{Jy}]} \\
\end{array}$ & $\begin{array}{l}m_{\mathrm{L}} \\
(4) \\
{[\%]} \\
\end{array}$ & $\begin{array}{c}\text { EVPA } \\
(5) \\
{[\mathrm{deg}]} \\
\end{array}$ \\
\hline $2015 / 05 / 31$ & $\begin{array}{c}97.5 \\
233.0 \\
343.5 \\
\end{array}$ & $\begin{array}{c}17.14 \pm 0.57 \\
9.82 \pm 0.33 \\
6.85 \pm 0.23 \\
\end{array}$ & $\begin{array}{l}0.6 \pm 0.3 \\
1.0 \pm 0.3 \\
0.3 \pm 0.3\end{array}$ & $\begin{array}{c}82.1 \pm 19.7 \\
-(29.1 \pm 12.1) \\
18.3 \pm 42.9\end{array}$ \\
\hline
\end{tabular}

Notes. The columns show (1) the observing epoch, (2) the central frequency, (3) the total flux, (4) the degree of the linear polarization and (5) the EVPA.

and was not resolved up to the baseline length of $537.5 k \lambda$ at all frequencies. Therefore, we constrained the source size to be $<1 /\left(537.5 \times 10^{3}\right) \mathrm{rad} \approx 0.38$ arcsecs. Table 3 summarizes the archival ALMA polarization measurements.

\subsection{Modelfitting, polarization measurements and their uncertainties}

In order to parameterize the size and the flux density of the VLBI core region in Stokes I, we made use of the MODELFIT procedure implemented in DIFMAP. We fitted circular Gaussians to the visibilities at each frequency and estimated their uncertainties following Schinzel et al. (2012). For the absolute flux density at $86 \mathrm{GHz}$, we conservatively adopted an uncertainty of $\sim 30 \%$ based on the limitation of the absolute flux calibration in this epoch.

The polarization components were identified by the local maxima of their linearly polarized intensities in the image plane (see Figs. 3 and 7). For each component, we defined a circular aperture whose diameter matched the mean beam size and was centered at the local peak of the polarized intensity. The properties of the polarized components - meaning the Stokes $I$ flux density $S$, the linearly polarized flux density $P$, the degree of linear polarization $m_{\mathrm{L}}$ and the EVPA $\chi$ - were then determined from the spatially integrated Stokes $I, Q$, and $U$ flux densities. Specifically, the integrated flux densities and the polarization parameters were computed using the following equations.

$$
\begin{aligned}
& Q_{\text {tot }}=\Sigma Q_{i, j} \times A_{\text {pixel }} / A_{\text {Beam }} \quad(\text { in Jy }), \\
& U_{\text {tot }}=\Sigma U_{i, j} \times A_{\text {pixel }} / A_{\text {Beam }} \quad(\text { in Jy }), \\
& S=\Sigma I_{i, j} \times A_{\text {pixel }} / A_{\text {Beam }} \quad \text { (in Jy), } \\
& P=\sqrt{Q_{\text {tot }}^{2}+U_{\text {tot }}^{2}} \quad \text { (in Jy), } \\
& m_{\mathrm{L}}=100 \times P / S \quad \text { (in percent }, \\
& \chi=0.5 \arctan \left(U_{\text {tot }} / Q_{\text {tot }}\right) \quad \text { (in radian), }
\end{aligned}
$$

where $I_{i, j}, Q_{i, j}$ and $U_{i, j}$ are the corresponding Stokes intensity values at each pixel $(i, j)$ in $\mathrm{Jybeam}^{-1}$ and $A_{\text {pixel }}$ and $A_{\text {Beam }}$ are the pixel and the beam area in square mas, respectively ${ }^{6}$. In these calculations, we also corrected for the Rice bias in the polarization intensity value at each pixel by following Wardle \& Kronberg (1974). We assumed that the uncertainty of $P$ was $30 \%$ at all frequencies. For $S$, we assumed $10 \%$ uncertainties at $\leq 43 \mathrm{GHz}$ (e.g., Lister \& Homan 2005; Jorstad et al. 2005)

\footnotetext{
6 We computed the area under the elliptical Gaussian beam, $A_{\text {Beam }}$, by $A_{\text {Beam }}=\pi \psi_{\text {maj }} \psi_{\text {min }} / 4 \ln 2$ where $\psi_{\text {maj,min }}$ are the FWHM of the elliptical Gaussian along the major and the major axis, respectively. The areas of individual pixels, $A_{\text {pixel }}$, are $0.01 \times 0.01,0.02 \times 0.02$ and $0.03 \times 0.03$ square mas at 86,43 and $15 \mathrm{GHz}$, respectively.
} 

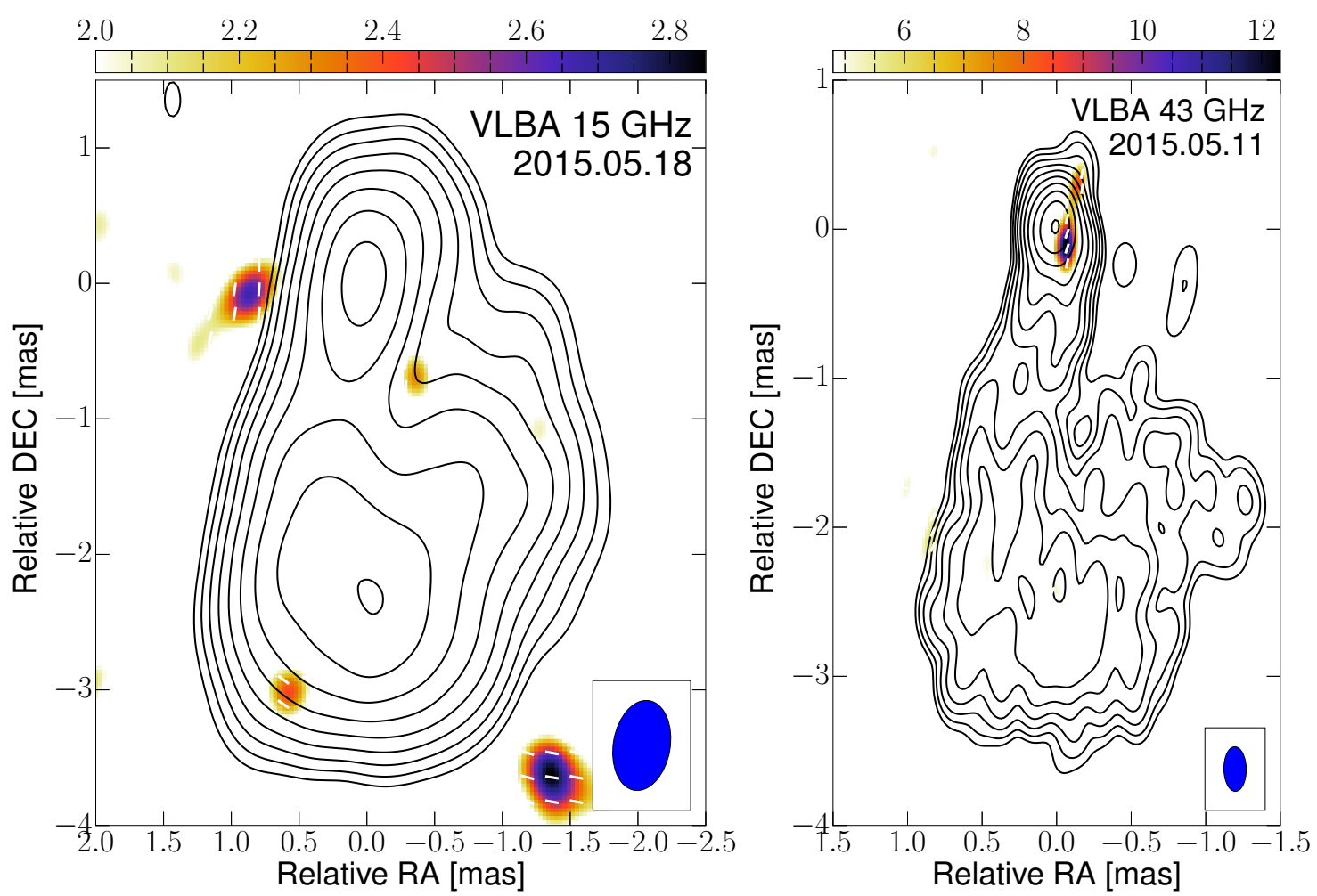

Fig. 2. Polarimetric VLBI images of $3 \mathrm{C} 84$ at 15 and $43 \mathrm{GHz}$. In both panels, the contours show the total intensity and increase by a factor of two from the lowest level. The color scale represents the polarized intensity (in mJybeam ${ }^{-1}$ ). The white bars in the images display the apparent EVPA. The blue ellipse in the bottom right corner shows the restoring beam. Left panel: VLBA $15 \mathrm{GHz}$ image. The contours start at $30 \mathrm{mJy}$ beam $^{-1}$. The color scale starts at $2.0 \mathrm{mJy}_{\text {beam }}{ }^{-1}$. Right panel: VLBA $43 \mathrm{GHz}$ image. The contours start at $7 \mathrm{mJy} \mathrm{beam}^{-1}$. The color scale starts at $4.8 \mathrm{mJy}$ beam $^{-1}$.

but $30 \%$ at $86 \mathrm{GHz}$ as explained before. The uncertainty of $m_{\mathrm{L}}$ was then obtained by propagating the errors of the integrated flux densities. The EVPA error was calculated as described in Sect. 2.1.2.

\section{Results}

\subsection{Total intensity structure and the core flux}

In Fig. 2 we show the quasi-simultaneous polarimetric VLBI images of 3C 84 at 15 and $43 \mathrm{GHz}$ in May 2015. The GMVA $86 \mathrm{GHz}$ images are shown in Fig. 3 with two different restoring beams in order to better illustrate both the extended and inner jet structure. The source clearly shows a limb-brightened jet at high frequencies ( $\geq 43 \mathrm{GHz}$ ). The limb-brightening is visible up to $\sim 2$ mas from the core. We made transverse cuts to the jet at a core separation $z \sim 0.4$ mas at 43 and $86 \mathrm{GHz}$ using the fullresolution image (Fig. 3, right) and show the intensity profiles of the slices in Fig. 4. The limb-brightened transverse structure is consistent with previous deep single-epoch (Nagai et al. 2014) and long-term (Jorstad et al. 2017) VLBA $43 \mathrm{GHz}$ observations of the source. At $86 \mathrm{GHz}$, the improved angular resolution resolves the edge-brightened structure down to $\sim 0.2$ mas core distance. Remarkably, within the inner 0.2 mas from the intensity peak, the nuclear region appears substantially resolved in the E-W direction and shows a complex morphology. The $86 \mathrm{GHz}$ core is well represented by a single circular Gaussian component whose FWHM size is comparable to the $43 \mathrm{GHz}$ core. However, the visibilities at long baselines $(\sim 1.0-2.5 G \lambda)$ suggest the presence of a more complex subnuclear structure (see Fig. 1). On larger scales, the overall source structure is quite similar to that shown in the $43 \mathrm{GHz}$ image (cf. Figs. 2 and 3), but the extended jet emission at larger core separations is overall fainter. A more detailed study of the total intensity structure is beyond the focus of this paper and will be presented elsewhere.

In Table 4 we show the flux densities $S_{\text {mod }}$ and FWHM sizes of the core at 15, 43 and $86 \mathrm{GHz}$ obtained with the MODELFIT procedure of DIFMAP. We fitted a single power-law to the core spectrum, that is $S \propto v^{+\alpha}$ where $v$ is the observing frequency and $\alpha$ is the spectral index. The results are shown in the left panel of Fig. 5. We find that the spectrum of the VLBI core is slightly inverted up to $86 \mathrm{GHz}$ with a spectral index of $\alpha=0.51 \pm 0.10$. We also show the time variation of the core flux at $43 \mathrm{GHz}$ in Fig. 6. In the same figure we also provide the peak values obtained with a circular Gaussian beam of 0.3 mas. The core brightens by $\sim 2$ Jy during 2015 and reaches a local maximum during June and July 2015.

\subsection{Linear polarization in the core region}

In Fig. 7 we show the linear polarization structure in the core region at 43 and $86 \mathrm{GHz}$ in more detail, restoring the maps with the VLBA $43 \mathrm{GHz}$ beam at both frequencies for better comparison. In May 2015, we detect linear polarization features in the core at high significance $\left(P I_{\text {peak }} / \sigma_{\mathrm{P}} \sim 7.0\right.$ and 5.5 at 43 and $86 \mathrm{GHz}$, respectively; see Table 1). At $15 \mathrm{GHz}$, no significant polarization features appear in the nuclear region. The polarization features at both 43 and $86 \mathrm{GHz}$ are slightly offset from the peak of the total intensity. The features P1 and P2 (i.e., $\mathrm{P} 2 \mathrm{a}+\mathrm{P} 2 \mathrm{~b}$ ) are separated by $\sim 0.2$ mas in the $\mathrm{E}-\mathrm{W}$ orientation, and could possibly be associated with the edges of the underlying limb-brightened jet seen at $22 \mathrm{GHz}$ by recent RadioAstron 

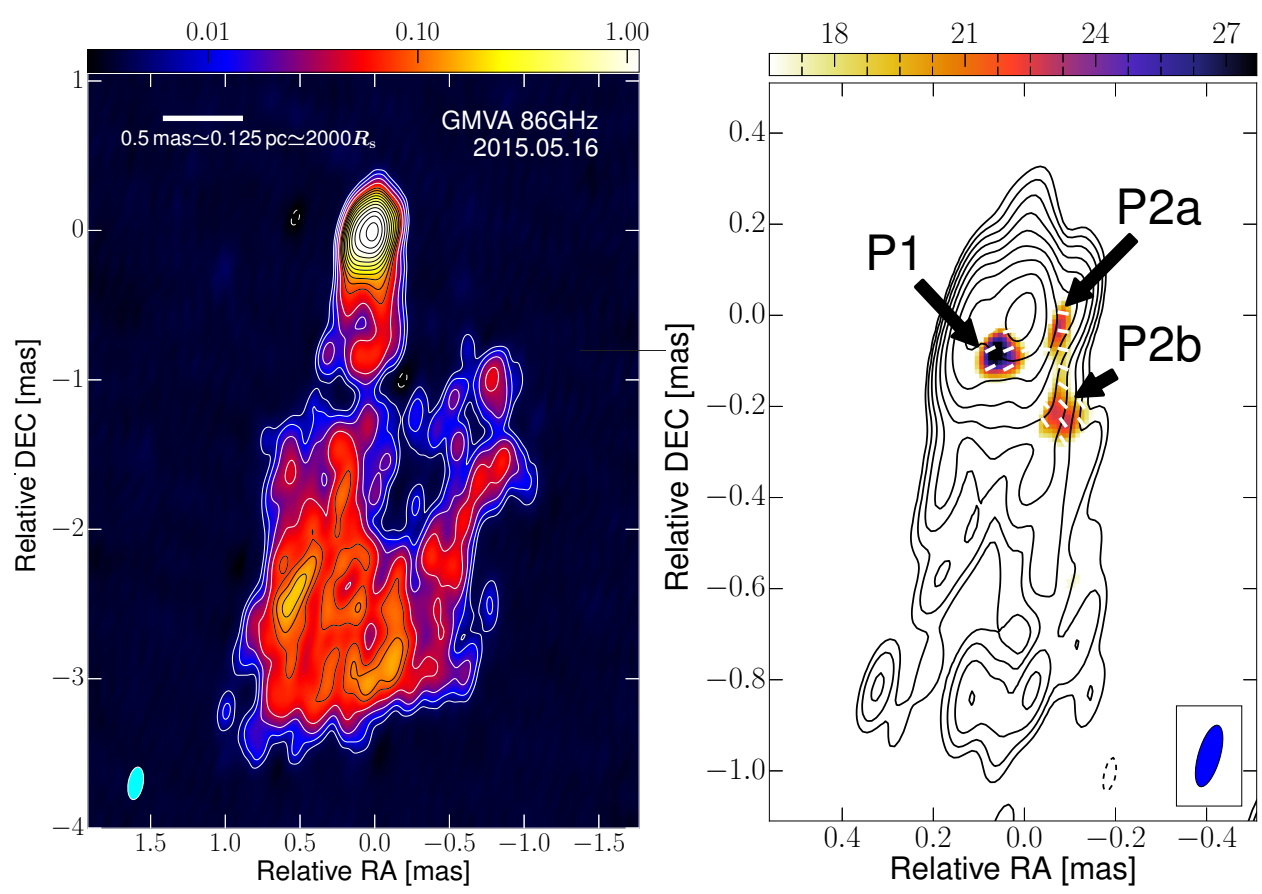

Fig. 3. GMVA $86 \mathrm{GHz}$ images of $3 \mathrm{C} 84$. In both panels, the contours show the total intensity and increase by a factor of two from the lowest level $\left(5 \mathrm{mJy}^{\mathrm{beam}} \mathrm{b}^{-1}\right)$. A negative contour of $-5 \mathrm{mJy}_{\text {beam }}^{-1}$ is also shown with dashed lines. Left panel: stokes $I$ image restored with an elliptical beam of $0.22 \times 0.10$ mas and position angle of $-10^{\circ}$. This beam is larger than the full resolution attainable with the GMVA. The peak of this image is $3.04 \mathrm{Jy}_{\text {beam }}^{-1}$. The cyan ellipse in the bottom-left corner illustrates the elliptical beam. The color scale represents the Stokes I pixel values in $\mathrm{Jy} \mathrm{beam}^{-1}$. The white bar and the text in the upper left corner denote the projected spatial scale of the image. Right panel: inner jet structure obtained with the full angular resolution of the GMVA. The color scale represents the linear polarization intensity and starts from $16.5 \mathrm{mJy}$ beam $^{-1}$.

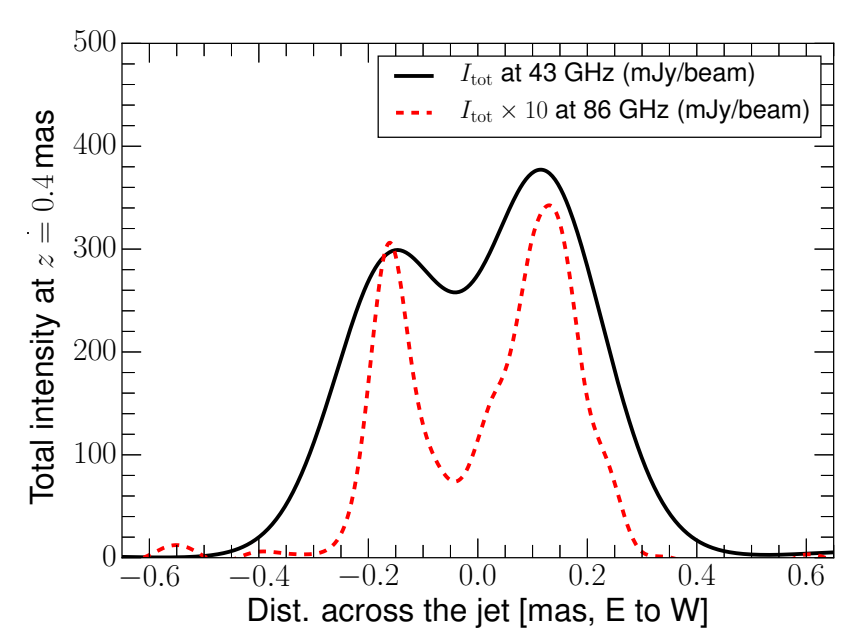

Fig. 4. Transverse jet intensity profiles at $43 \mathrm{GHz}$ (black solid line) and $86 \mathrm{GHz}$ (red broken line) obtained at 0.4 mas core distance as a function of distance across the jet (increasing toward west).

observations (e.g., Giovannini et al. 2018). The P2 component is present in both maps at similar locations. However, the P1 component in the $86 \mathrm{GHz}$ image has no clear counterpart at $43 \mathrm{GHz}$. Using the pixel values at the position of P1, we estimate its $3 \sigma_{\mathrm{P}}$ upper limit to $m_{\mathrm{L}}$ to be $\lesssim 0.2 \%$ at $43 \mathrm{GHz}$. At $43 \mathrm{GHz}$, there exists another polarized feature $\left(\sim 8 \mathrm{mJy} \mathrm{beam}^{-1}\right)$ at a separation of $\sim 0.3$ mas to the north of the core, perhaps associated with a counterjet. We searched the VLBA-BU-BLAZAR database for evidence in support of significant polarization north of the main intensity peak in several epochs. While the polarization detection close to the total intensity peak can be seen in many epochs, we found polarization north of it only in a few epochs. Therefore we do not find strong evidence for a persistent polarization on the counterjet side. Except for this faint northern polarization feature, the $43 \mathrm{GHz}$ VLBA polarization images in the other epochs show similar linear polarization flux densities in the core region, although the relative position of the polarization peak seems to change with time (see Appendix C).
Table 4. VLBI core properties in May 2015.

\begin{tabular}{|c|c|c|}
\hline $\begin{array}{c}v_{\mathrm{obs}} \\
(1) \\
{[\mathrm{GHz}]}\end{array}$ & $\begin{array}{c}S_{\text {mod }} \\
(2) \\
{[\mathrm{Jy}]}\end{array}$ & $\begin{array}{c}F W H M \\
(3) \\
{[\mathrm{mas}]}\end{array}$ \\
\hline 86 & $5.6 \pm 1.7$ & $0.13 \pm 0.04$ \\
\hline 43 & $4.8 \pm 0.7$ & $0.14 \pm 0.01$ \\
\hline 15 & $2.6 \pm 0.4$ & $0.27 \pm 0.02$ \\
\hline
\end{tabular}

Notes. The columns show (1) the central observing frequency and (2),(3) the total flux and the FWHM size of the VLBI component obtained by the Gaussian model fitting.

At $86 \mathrm{GHz}$, the degrees of the linear polarization $m_{\mathrm{L}}$ of the polarized components are in the range of $\sim 3-6 \%$ when convolved with the small GMVA observing beam. The $86 \mathrm{GHz} m_{\mathrm{L}}$ values decrease to $\sim 1-2 \%$ when convolved with the VLBA $43 \mathrm{GHz}$ beam. For the $\mathrm{P} 2$ component, the $m_{\mathrm{L}}$ value is significantly lower at $43 \mathrm{GHz}(\sim 0.4-0.5 \%$ in this epoch). At $15 \mathrm{GHz}$, the nondetection suggests a $3 \sigma$ upper limit to $m_{\mathrm{L}}$ of $\lesssim 0.1 \%$, which is in agreement with the results of Taylor et al. (2006). In order to minimize the effect of the different angular resolutions of the VLBA and the GMVA images, we now determine the polarization properties convolving the maps with the same VLBA $43 \mathrm{GHz}$ beam (Fig. 7) and compare the results in the following analysis. Table 5 provides a summary of the properties of the polarized components in May 2015 (see Appendix C for results at $43 \mathrm{GHz}$ at other epochs).

In order to investigate the frequency dependence of the linear polarization of the core region, we combined linear polarization measurements from the literature with the data from this paper. The results are shown in the right panel of Fig. 5. The linear polarization of the core clearly shows an increasing trend with frequency, which can be represented by a powerlaw fit $m_{\mathrm{L}} \propto v^{0.77 \pm 0.18}$, in which we have combined all available $m_{\mathrm{L}}$ measurements. We note that the index of the power law could be affected by some systematic uncertainties due to the different angular resolutions of the telescopes. Nevertheless, we 

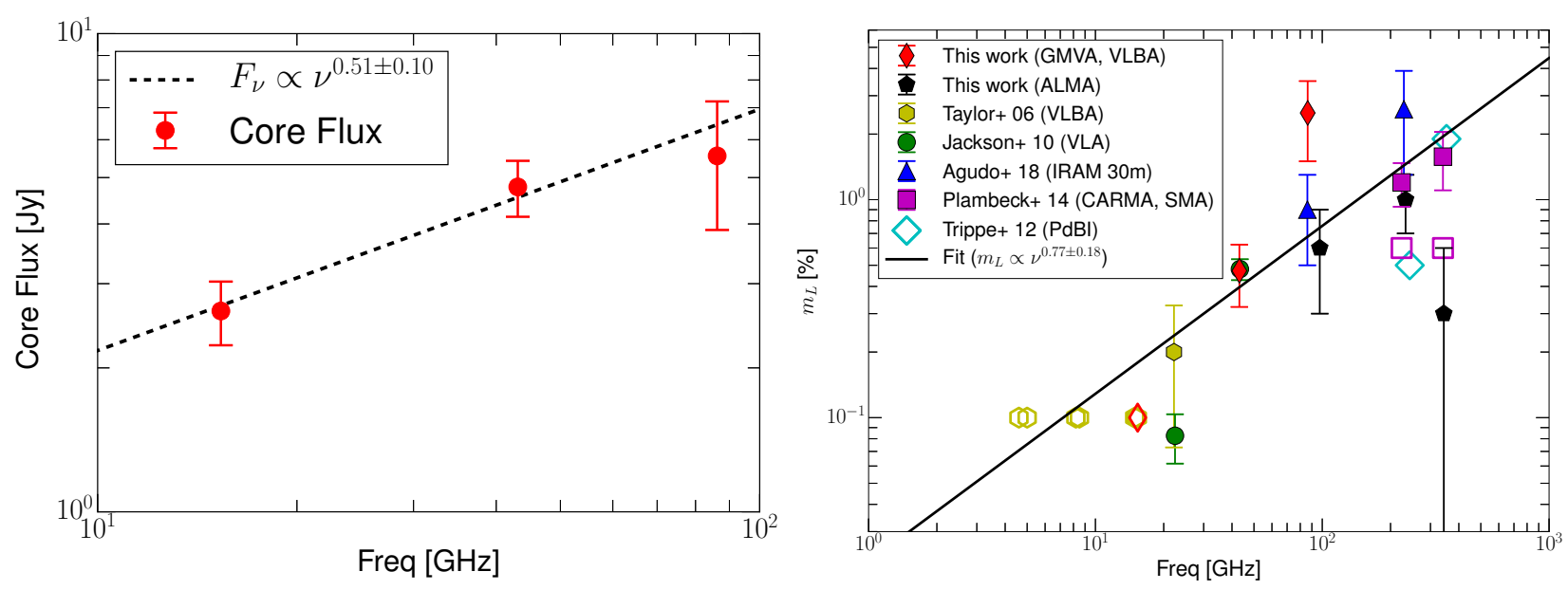

Fig. 5. Left panel: total intensity spectrum of the VLBI core region between 15 and $86 \mathrm{GHz}$. Right panel: degree of linear polarization in the VLBI core region obtained from this work and previous VLBA observations (Taylor et al. 2006) and integrated values from the literature (Jackson et al. 2010; Trippe et al. 2012; Plambeck et al. 2014; Agudo et al. 2018b). All the VLBI polarization measurements were made from the pixel values near the peak of the Stokes $I$. Filled and open symbols of the same marker indicate measured values and upper limits, respectively. Citations and corresponding instruments are shown in the legend. We note that at $230 \mathrm{GHz}$ only upper limits were observed before May 2011 (Trippe et al. 2012 ; Plambeck et al. 2014). After October 2011, Plambeck et al. (2014) report a significantly higher linear polarization degree.

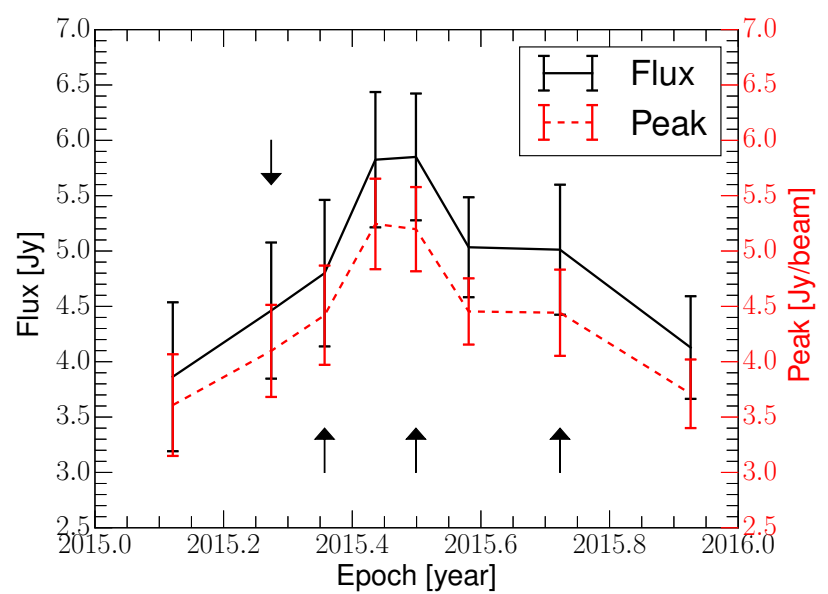

Fig. 6. VLBA $43 \mathrm{GHz}$ core flux and the peak values during 2015. Arrows indicate epochs during which reliable polarization has been detected.

can clearly see that both VLBI and non-VLBI measurements show an increase of linear polarization degree toward higher frequencies.

\subsection{Rotation measure between 43 and $344 \mathrm{GHz}$ in May 2015}

We determine the RM associated with the Faraday rotation by measuring the variation of the observed EVPA with frequency. When a linearly polarized wave at a wavelength $\lambda$ passes through magnetized plasma, the intrinsic EVPA of the emission $\chi_{\text {int }}$ is rotated and the observed EVPA $\chi(\lambda)$ is

$\chi(\lambda)=\chi_{\text {int }}+\mathrm{RM} \times \lambda^{2}$.

The RM is determined by the gradient of $\chi(\lambda)$ versus $\lambda^{2}$ after solving for the $n \pi$ ambiguity in the angle. To resolve the $n \pi$ ambiguity, we assume that most of the polarized flux observed by the ALMA at $97.5,233.0$ and $343.5 \mathrm{GHz}$ originates from the same VLBI core region. This assumption appears to be reasonable based on the flat spectrum of the linearly polarized flux density between 97.5 and $233.0 \mathrm{GHz}$ measured by ALMA $(\sim 100 \mathrm{mJy}$ at both frequencies; see Table 3 ). We then compute the integrated Stokes $Q$ and $U$ flux densities of the VLBI core region at 43 and $86 \mathrm{GHz}$ to obtain the spatially integrated EVPAs. The spatially integrated EVPAs within the $43 \mathrm{GHz}$ band are shown in Table 5. At $86 \mathrm{GHz}$, the integrated EVPA is $\chi=-(70 \pm 16)$ deg. Subsequently, we compare all these EVPAs simultaneously to find a best $\lambda^{2}$ fit.

We resolve the $n \pi$ ambiguity by following Hovatta et al. (2012). The authors determined the smallest possible EVPA rotations at each frequency to achieve a statistically acceptable fit with the $\lambda^{2}$ model. Similarly, we rotated the 86.0, 97.5, 233.0, and $343.5 \mathrm{GHz}$ EVPAs by $\left(n_{1}, n_{2}, n_{3}, n_{4}\right) \times \pi$, respectively, where $n_{i} \in(-30,-29, \ldots, 29,30)$ and $i \in(1,2,3,4)$. We then calculated corresponding $\chi^{2}$ values for each rotation. We note that $n=30$ corresponds to $\mathrm{RM} \sim 10^{8} \mathrm{rad} \mathrm{m}^{-2}$ for the frequency range considered here. The RM of $\sim 10^{8} \mathrm{rad} \mathrm{m}^{-2}$ is also equivalent to a single $180^{\circ}$ wrap within the $43 \mathrm{GHz}$ band. Therefore, we are assuming that there is no $\mathrm{RM} \gtrsim 10^{8} \mathrm{rad} \mathrm{m}^{-2}$ in the source (cf. Plambeck et al. 2014). For the same reason, we did not apply the $n \pi$ rotation within the $43 \mathrm{GHz}$ band. We then chose a set of $n_{i}$ which provided the minimum $\chi^{2}$ value.

We find a best $\lambda^{2}$ fit with $n_{1}=-2, n_{2}=-3, n_{3}=-3$ and $n_{4}=$ -3 (corresponding $\chi^{2}=3.52$ and the reduced $\chi^{2}=0.59$; see Fig. 8). Accordingly, we obtain $\mathrm{RM}=(2.02 \pm 0.03) \times 10^{5} \mathrm{rad} \mathrm{m}^{-2}$ and $\chi_{\text {int }}=-(578 \pm 7) \mathrm{deg}$ (or equivalently $\chi_{\text {int }}=-(38 \pm 7) \mathrm{deg}$ ). We note this RM is comparable to $\mathrm{RM} \sim 9 \times 10^{5} \mathrm{rad} \mathrm{m}^{-2}$ previously reported by Plambeck et al. (2014).

\subsection{Rotation measure within the $43 \mathrm{GHz}$ band}

We applied the same RM fitting procedure to the multiepoch VLBA $43 \mathrm{GHz}$ data using the EVPAs measured at the four different IFs. We tested the significance of the EVPA rotation within the $43 \mathrm{GHz}$ band for $3 \mathrm{C} 84$ by performing the same analysis to the core regions of two comparison sources $0235+164$ and $3 \mathrm{C} 279$, which were observed in the same epochs by the VLBA-BU-BLAZAR monitoring program. The instrumental polarization calibration and the sub-band EVPA calibration for the two sources were completely identical to those of 3C 84 during 2015. Therefore, we expect that the EVPA change within 


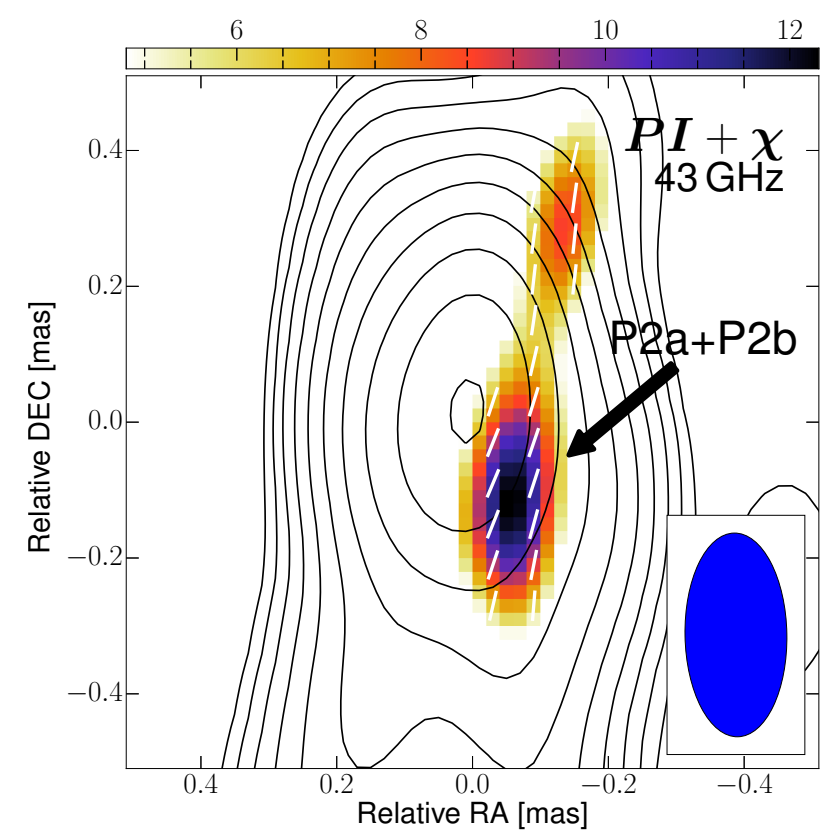

(a)

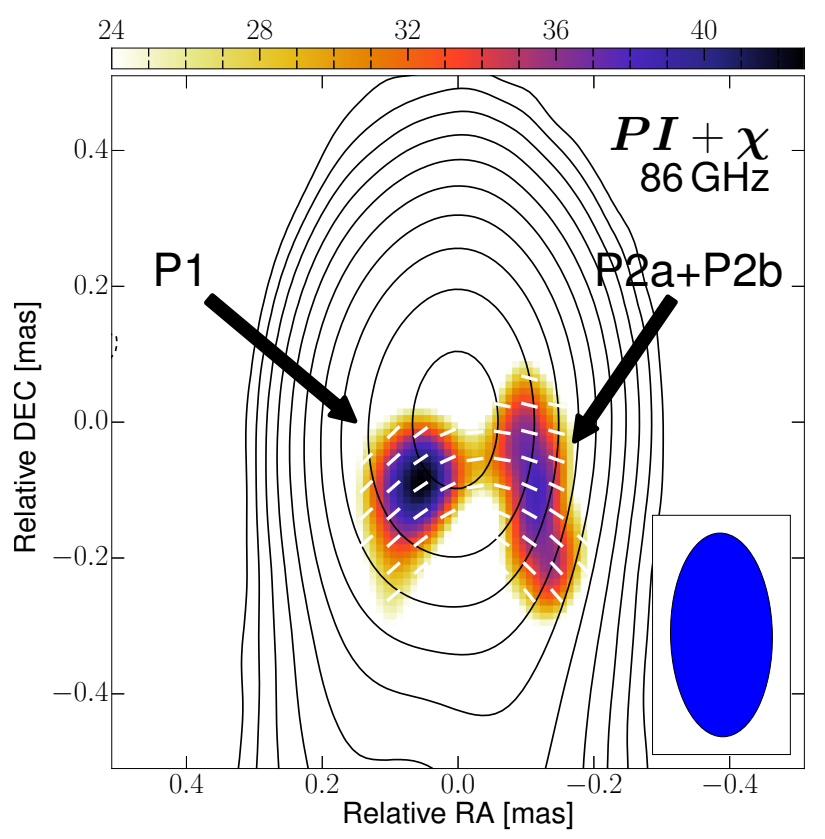

(b)

Fig. 7. Comparison of the total intensity, the polarized intensity $(P I)$ and the polarization angle $(\chi)$ in the nuclear region at 43 and $86 \mathrm{GHz}$. The maps are restored with the same VLBA $43 \mathrm{GHz}$ CLEAN beam and displayed over the same RA and Dec ranges ( \pm 0.5 mas). The arrows show the polarized component identifications. Panel $a$ : as in the central panel of Fig. 2 (43 GHz) but zoomed into the core. Panel b: GMVA $86 \mathrm{GHz}$ total and linear polarization image restored with the VLBA $43 \mathrm{GHz}$ beam. The contours start at $6 \mathrm{mJy} \mathrm{beam}^{-1}$ and increase by a factor of two from the lowest level. The color scale starts from $24 \mathrm{mJy}_{\text {beam }}{ }^{-1}$.

Table 5. Properties of the polarized components in May 2015.

\begin{tabular}{ccccc}
\hline \hline $\begin{array}{c}v_{\text {obs }} \\
(1)\end{array}$ & $\begin{array}{c}\text { ID } \\
{[2)}\end{array}$ & $\begin{array}{c}S_{v} \\
(3)\end{array}$ & $\begin{array}{c}m_{v} \\
(4) \\
{[\%]}\end{array}$ & $\begin{array}{c}\chi_{v} \\
(5) \\
{[\mathrm{GHz}]}\end{array}$ \\
\hline 86.252 & $\mathrm{P} 1$ & $0.70 \pm 0.21$ & $3.4 \pm 1.4$ & $-(54 \pm 17)$ \\
& $\mathrm{P} 1^{a}$ & $1.5 \pm 0.4$ & $1.4 \pm 0.6$ & $-(43 \pm 17)$ \\
& $\mathrm{P} 2 \mathrm{a}$ & $0.27 \pm 0.08$ & $6.3 \pm 2.7$ & $-(89 \pm 17)$ \\
& $\mathrm{P} 2 \mathrm{~b}$ & $0.05 \pm 0.02$ & $44 \pm 20^{b}$ & $47 \pm 17^{b}$ \\
& $\mathrm{P} 2 \mathrm{a}+\mathrm{P} 2 \mathrm{~b}^{a}$ & $1.3 \pm 0.4$ & $2.2 \pm 0.9$ & $71 \pm 17$ \\
\hline 43.0075 & $\mathrm{P} 2 \mathrm{a}+\mathrm{P} 2 \mathrm{~b}^{2}$ & $2.0 \pm 0.2$ & $0.44 \pm 0.14$ & $-(9 \pm 10)$ \\
43.0875 & & & $0.49 \pm 0.16$ & $-(20 \pm 10)$ \\
43.1515 & & & $0.53 \pm 0.17$ & $-(24 \pm 10)$ \\
43.2155 & & & $0.43 \pm 0.13$ & $-(15 \pm 10)$ \\
\hline 15.352 & & N/A & $<0.1^{c}$ & N/A \\
\hline
\end{tabular}

Notes. The columns show (1) the central frequency, (2) the component identification, (3) the Stokes I flux density, (4) the degree of linear polarization and (5) the EVPA. The Stokes $I$ flux densities are consistent at the four separate frequencies within the $43 \mathrm{GHz}$ band and we omit the numbers for the other three $43 \mathrm{GHz}$ sub-bands. ${ }^{(a)}$ Obtained from the image in panel (B) of Fig. 7. ${ }^{(b)}$ The component is associated with the outer edge of the jet and could have higher systematic uncertainties than our estimation. ${ }^{(c)} 3 \sigma$ upper limit from the nondetection.

the $43 \mathrm{GHz}$ band will be systematically the same for the three sources if the EVPA changes are dominated by residual calibration errors.

In Fig. 9 we show the EVPAs $\chi$ for $0235+164$ and $3 C 279$, with respect to their mean values, $\langle\chi\rangle$. Within the $43 \mathrm{GHz}$ band, the EVPA of $3 C 279$ varies only by $\leq 6 \mathrm{deg}$ in all epochs $(\leq 8 \mathrm{deg}$ for $0235+164$ ). This is insignificant compared to our presumed ten-degree angle uncertainty at $43 \mathrm{GHz}$. In addition, the two

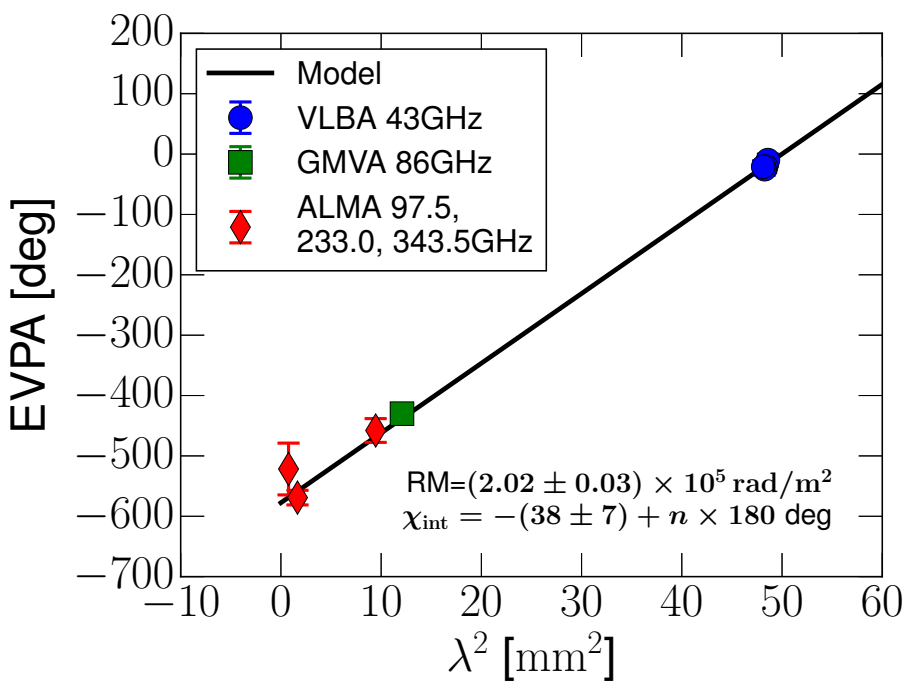

Fig. 8. Spatially integrated EVPA vs. the squared wavelength $\lambda^{2}$ (error bars) and the best $\lambda^{2}$ fit (solid line) in May 2015.

sources show strongly correlated EVPA offset from their mean values in all epochs. From this we determine that the $43 \mathrm{GHz}$ inband $\mathrm{RM}$ values smaller than $\sim(2-3) \times 10^{5} \mathrm{rad} \mathrm{m}^{-2}$ are insignificant against the residual calibration errors.

After this consideration, we show the EVPAs measured from the core of $3 \mathrm{C} 84$ at the $43 \mathrm{GHz}$ band in Fig. 10. The corresponding RM values obtained from the fitting are shown in Table 6 and a plot of the RM versus time is given in Fig. 11. In contrast to $0235+164$ and 3 C 279, 3C 84 shows much larger EVPA variation within the $43 \mathrm{GHz}$ band $(15-21 \mathrm{deg})$. This EVPA variation with wavelength is larger than the residual calibration errors that we estimated. This results in a significant RM 


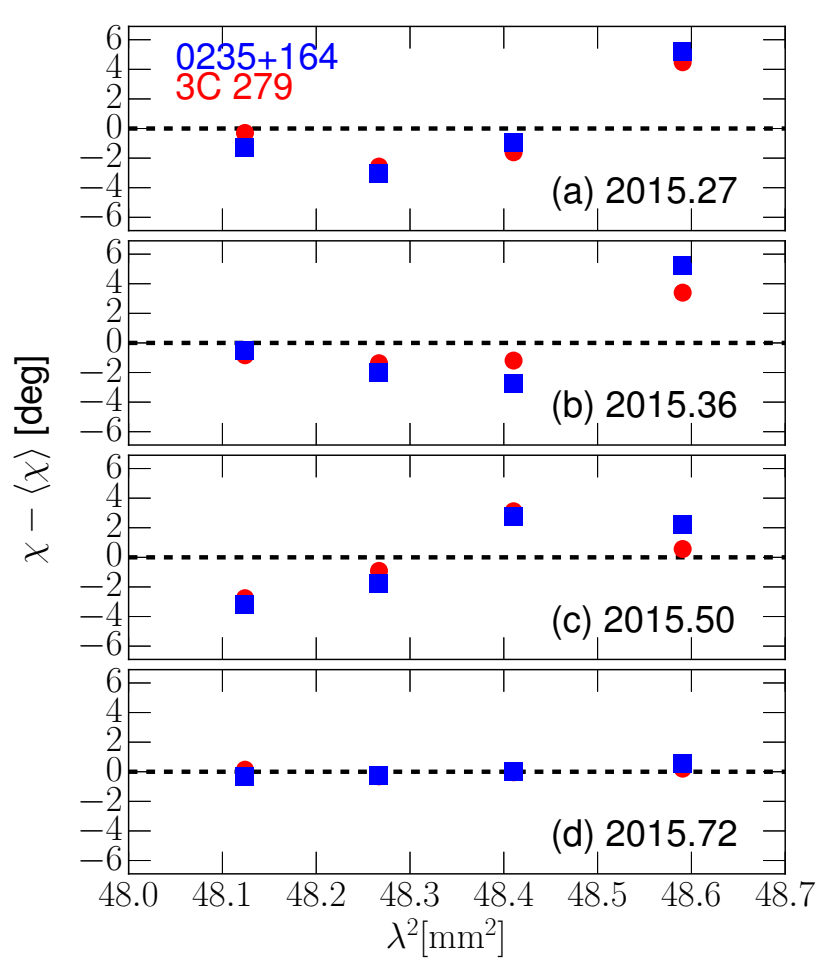

Fig. 9. Variation of the EVPA in each sub-band, $\chi$, with respect to the mean EVPA, $\langle\chi\rangle$. Blue squares and red circles are for $0235+164$ and 3 C 279, respectively. Error bars are omitted for clarity. We note the strong correlation between the two sources.

$\left(|\mathrm{RM}| \gtrsim 5 \times 10^{5} \mathrm{rad} \mathrm{m}^{-2}\right.$ for three out of four epochs). Accordingly, we consider the large RM obtained for 3C 84 within the $43 \mathrm{GHz}$ band to be intrinsic to the source. We also note a discrepancy in the form of a negative RM (i.e., decreasing EVPA value with increasing $\lambda^{2}$ ), which is present only in Apr 2015. We note that the EVPAs of the calibrators in this epoch generally increase with increasing $\lambda^{2}$ (see the top panel of Fig. 9). If the systematic EVPA rotation in the calibrators were purely due to residual calibration errors, the intrinsic RM of $3 \mathrm{C} 84$ in this epoch would become even more negative.

\section{Discussion}

\subsection{Polarization structure}

Here we briefly discuss the possible implications of the polarization structure in the core region. At $86 \mathrm{GHz}$, the two subnuclear polarization features, $\mathrm{P} 1$ and $\mathrm{P} 2$, have different degrees of linear polarization. In order to highlight the polarization asymmetry, we chose the $86 \mathrm{GHz}$ image shown in Fig. 7 and made a transverse cut to the core region (in the $\mathrm{E}-\mathrm{W}$ direction at $\sim 0.1$ mas downstream of the jet). From this slice, we obtained transverse total and polarization intensity profiles (Fig. 12).

A transverse polarization asymmetry in the core region has occasionally been seen in several other AGNs (Gómez et al. 2008, 2016; O'Sullivan \& Gabuzda 2009; Clausen-Brown et al. 2011). This is often related to the geometry of the jet (e.g., jet inclination and opening angle) and the magnetic field (e.g., pitch angle; see Lyutikov et al. 2005; Clausen-Brown et al. 2011; Porth et al. 2011). We note that the intrinsic and spatially integrated EVPA is $\chi_{\text {int }} \sim-38 \mathrm{deg}$ in May 2015. This angle is neither parallel nor perpendicular to the position angle of the inner jet ( $\sim 170$ deg inferred from Fig. 7$)$, suggesting that the orientation of the magnetic field might be oblique to the jet.

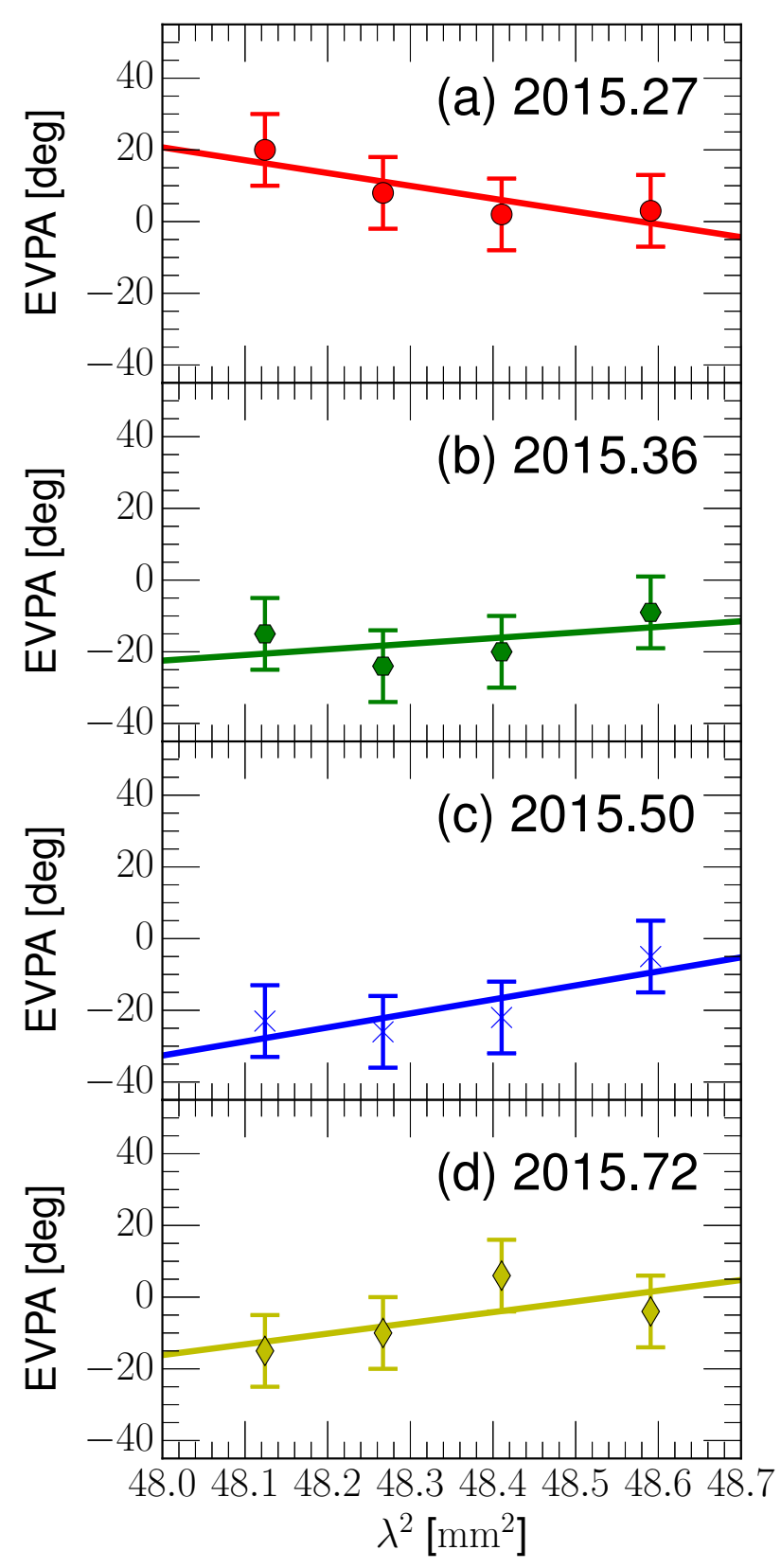

Fig. 10. EVPA of $3 C 84$ vs. the squared wavelength $\lambda^{2}$ within the VLBA $43 \mathrm{GHz}$ band (error bars) and the best-fit Faraday rotation model (solid line).

However, we also note that the polarization structure is also highly time-variable at $43 \mathrm{GHz}$ during 2015 when the VLBI core flux density showed a small outburst. Such outbursts often accompany structural changes within the jet - for example ejection of new VLBI components from the core - and also likely lead to changes in the polarized morphology near the core. In fact, the peak of the linear polarization in the VLBA $43 \mathrm{GHz}$ images moves with respect to the peak of the total intensity by $\sim 0.2$ mas on an approximately monthly timescale, especially in the $\mathrm{E}-\mathrm{W}$ direction (see Appendix $\mathrm{C}$ ). We note that the typical VLBA beam at $43 \mathrm{GHz}$ beam is $\sim 0.15$ mas in $\mathrm{E}-\mathrm{W}$ direction. Thus the position offset of $\sim 0.2$ mas is larger than the beam size and should be significant. Single-dish monitoring observations of 3C 84 at millimeter wavelengths by Agudo et al. (2018b) also show polarization time-variability on longer timescales. Therefore, one cannot rule out the possibility of turbulence 
Table 6. RM within the VLBA $43 \mathrm{GHz}$ band.

\begin{tabular}{ccc}
\hline \hline $\begin{array}{c}\text { Epoch } \\
\text { [yyyy/mm/dd] }\end{array}$ & $\begin{array}{c}\mathrm{RM} \\
{\left[\times 10^{5} \mathrm{rad} \mathrm{m}^{-2}\right]}\end{array}$ & $\begin{array}{c}\chi \\
{[\mathrm{deg}]}\end{array}$ \\
\hline $2015 / 04 / 12$ & $-(6.2 \pm 2.6)$ & $11 \pm 5$ \\
$2015 / 05 / 11$ & $2.7 \pm 3.5$ & $-(14 \pm 5)$ \\
$2015 / 07 / 02$ & $6.8 \pm 3.3$ & $-(11 \pm 5)$ \\
$2015 / 09 / 22$ & $5.2 \pm 4.2$ & $-(8 \pm 5)$ \\
\hline
\end{tabular}

Notes. From left to right, columns denote the observing epoch, the RM and the EVPA interpolated to the central frequency of the $43 \mathrm{GHz}$ band $(43.1155 \mathrm{GHz})$.

(e.g., Marscher 2014) or varying opacity across the jet which may also produce similar asymmetric and variable polarization structures.

\subsection{Possible explanations for the polarization spectrum and EVPA rotation}

In the rest of this discussion, we focus on possible physical explanations for the observed frequency dependence of the fractional linear polarization and EVPA. Frequency dependence of the polarization properties can be explained by several scenarios. For instance, the inverted total intensity spectrum (Fig. 5, left) between 15 and $86 \mathrm{GHz}$ suggests high opacity and large synchrotron self-absorption. In such jets, the emission at a higher frequency originates from a region closer to the base of the jet (e.g., Lobanov 1998). If entanglement of the magnetic field were the main source for the low linear polarization at low frequencies, a larger polarization degree $m_{\mathrm{L}}$ at higher frequencies could be interpreted as progressively more ordered magnetic fields in the inner jet region (e.g., Agudo et al. 2014, 2018b). Alternatively, the large synchrotron opacity in the core itself can transform the observed polarization, causing the linear polarization to increase with decreasing opacity (i.e., toward shorter wavelengths; see Pacholczyk 1970; Jones \& Odell 1977). In particular, the transition from optically thick to optically thin is accompanied by a 90 deg flip of the EVPA (Pacholczyk 1970).

We point out, however, that the impact of Faraday rotation is also significant because Faraday depolarization is a sensitive function of the observing frequency (Burn 1966; Sokoloff et al. 1998). Certainly, Faraday rotation may be not be a unique explanation of the large EVPA rotations across the observing frequencies. However, we also find large EVPA rotation and the corresponding RM within the $43 \mathrm{GHz}$ band (Fig. 10). This suggests that opacity effects alone cannot explain the results because the opacity change within the $43 \mathrm{GHz}$ band is most likely small given the small fractional bandwidth of $256 \mathrm{MHz} / 43 \mathrm{GHz} \sim 6 \times 10^{-3}$.

Within this perspective, the spectrum of the circular polarization in 3C 84 is also noteworthy. The inner jet of 3C 84 has a large degree of circular polarization $m_{\mathrm{C}} \sim 1-3 \%$ at 15-22 GHz (Homan \& Wardle 2004). At higher frequencies, there are still no direct VLBI measurements of the circular polarization in this source. However, single-dish observations suggest that the fractional circular polarization has a flat spectrum between centimeter and millimeter wavelengths $\left(m_{\mathrm{C}} \sim\right.$ a few $\times 0.1 \%$ at both $5-8 \mathrm{GHz}$ and $86-230 \mathrm{GHz}$; Aller et al. 2003; Myserlis et al. 2018; Agudo et al. 2010; Thum et al. 2018). As these authors suggest, the combination of low fractional linear polarization and high circular polarization is presumably produced by Faraday conversion, which is accompanied by Faraday rotation in inhomogeneous plasma that is located within (or per-

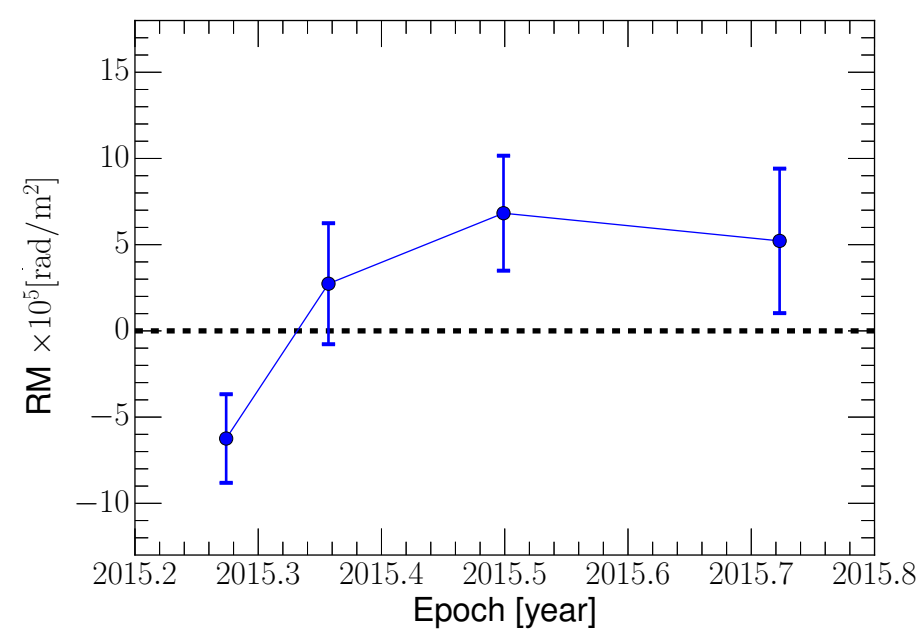

Fig. 11. Time variation of the $43 \mathrm{GHz}$ sub-band $\mathrm{RM}$ for $3 \mathrm{C} 84$.

haps external to) the jet (Jones \& Odell 1977; Wardle \& Homan 2003; MacDonald \& Marscher 2018). Therefore, we investigate in the following the impact of Faraday rotation in terms of Faraday depolarization for different types of Faraday screens.

\subsection{Faraday depolarization}

Based on the previous discussion (Sect. 4.2), we now discuss the impact of the Faraday effect assuming different types of Faraday screens. For the central VLBI core region of $3 \mathrm{C} 84$, an external Faraday screen can be located at various places. For example, a free-free absorption disk (Walker et al. 2000), which might be clumpy (Fujita \& Nagai 2017), could act as a Faraday screen. Another possibility is the accretion flow, which surrounds the central engine (Plambeck et al. 2014). We note that a radiatively inefficient accretion flow (RIAF) has been used to interpret the frequency dependence of the linear and circular polarization of Sgr A* (e.g., Bower et al. 2002; Muñoz et al. 2012), which resembles our observations of $3 \mathrm{C} 84$. Finally, the host galaxy of 3C 84 (NGC 1275) contains a substantial amount of intergalactic gas, whose effect is seen on the parsec-scale jet polarization at $\lesssim 22 \mathrm{GHz}$ (Taylor et al. 2006).

In the following we model the Faraday depolarization for three very simple cases. We then discuss their physical implications in subsequent Sects. 4.3.1 and 4.3.2. We assume three types of Faraday screens:

1. A foreground screen with a disordered magnetic field and random RM fluctuations;

2. A foreground screen with an ordered magnetic field and a smooth RM gradient within the observing beam; and

3. A Faraday depolarization inside the jet and a uniform magnetic field.

Following Burn (1966), the observed degree of linear polarization $m_{\mathrm{obs}}$ is a function of the observing wavelength, which for the three above cases can be written as:

Case I : $\quad m_{\mathrm{obs}}=m_{0} \exp \left(-2 \sigma^{2} \lambda^{4}\right)$,

Case II : $\quad m_{\mathrm{obs}}=m_{0}\left|\frac{\sin \left(\Delta \mathrm{RM} \lambda^{2}\right)}{\Delta \mathrm{RM} \lambda^{2}}\right|$,

Case III : $\quad m_{\mathrm{obs}}=m_{0}\left|\frac{\sin \left(2 \mathrm{RM} \lambda^{2}\right)}{2 \mathrm{RM} \lambda^{2}}\right|$,

where $m_{0}$ is the intrinsic linear polarization degree, $\lambda$ is the observing wavelength, $\sigma$ is the standard deviation of the 


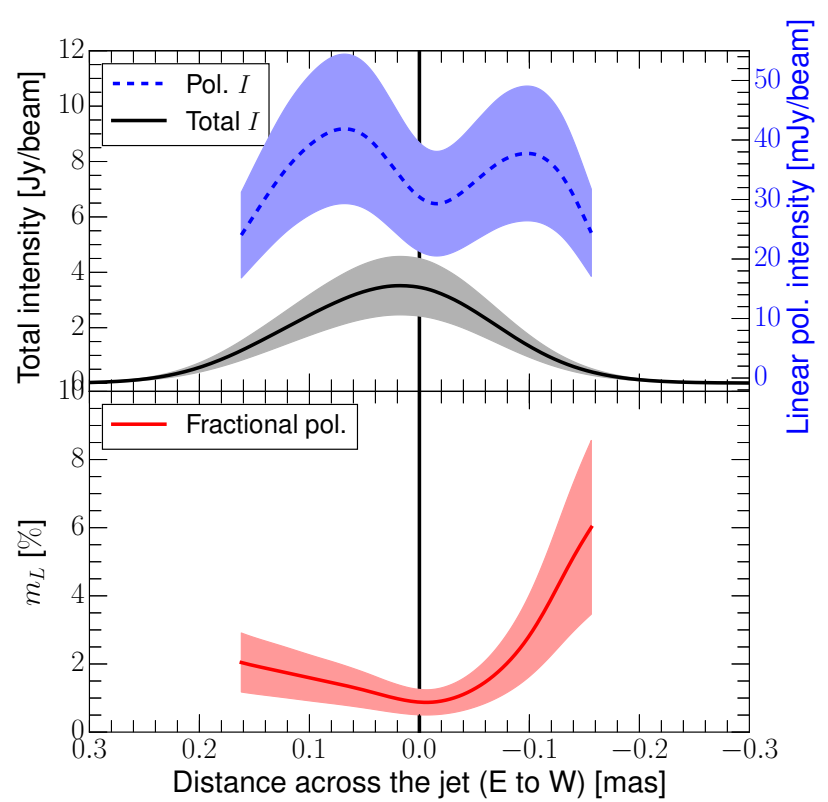

Fig. 12. Top panel: total intensity, linear polarization intensity; bottom panel: degree of linear polarization at $86 \mathrm{GHz}$ obtained by the slice transverse to the core and calculated by the pixel values in the image plane (negative core separation toward west; see Fig. 7). The solid black line indicates the position of the total intensity peak. The shaded area marks the measurement errors, assuming 30\% uncertainty in the flux density measurements at $86 \mathrm{GHz}$ (see Table 4). Only linear polarization higher than $24 \mathrm{mJy}^{\text {beam }}{ }^{-1}$ is shown.

dispersion of the RM in the external Faraday screen, $\Delta \mathrm{RM}$ is the RM gradient within the beam and RM is the observed rotation measure. For Case I, we assume $\sigma \approx \epsilon \times \mathrm{RM}$ where $\epsilon$ is a scaling factor describing the level of the RM dispersion (i.e., larger $\epsilon$ for more inhomogeneity). We start the calculation with $\epsilon=1$. Similarly, we assume $\Delta \mathrm{RM} \sim \mathrm{RM}$ for Case II. For Cases II and III, we have conservative upper limits for $m_{\mathrm{obs}}$ by $m_{\mathrm{obs}} \leq m_{0} /\left(\Delta \mathrm{RM} \lambda^{2}\right)$ and $m_{\mathrm{obs}} \leq m_{0} /\left(2 \mathrm{RM} \lambda^{2}\right)$, respectively. We choose $m_{0}=10 \%$, which is the theoretical maximum value in the case of high synchrotron opacity (Pacholczyk 1970). Finally, we take a $\mathrm{RM} \sim 9 \times 10^{5} \mathrm{rad} \mathrm{m}^{-2}$ from Plambeck et al. (2014), which is also close to the largest RM value found in our work $\left(\sim 7 \times 10^{5} \mathrm{rad} \mathrm{m}^{-2}\right)$. We then compare the upper limits with the observed $m_{\mathrm{L}}$ values in May 2015. For the P2 component, we use the $m_{\mathrm{L}}$ values at 86 and $43 \mathrm{GHz}$ of Table 5 and adopt $m_{\mathrm{L}} \lesssim 0.1 \%$ as an upper limit at $15 \mathrm{GHz}$. For the P1 component, we take the measured $m_{\mathrm{L}}$ value at $86 \mathrm{GHz}$ and adopt $m_{\mathrm{L}} \lesssim 0.2 \%$ and $\lesssim 0.1 \%$ as upper limits for 43 and $15 \mathrm{GHz}$, respectively. For both components, we use the polarization degrees as measured with the VLBA $43 \mathrm{GHz}$ restoring beam.

In Fig. 13 we show the upper limits for the linear polarization degree calculated for the three depolarization models. The plots reveal several important points. In Case I (red lines), the RM dispersion $\sigma$ is as large as $9 \times 10^{5} \mathrm{rad} \mathrm{m}^{-2}$ for $\epsilon \sim 1$. This is apparently problematic for both P2 and P1 because of the strong depolarization (solid red lines). We find that $\sigma \sim 2.5 \times$ $10^{4} \mathrm{rad} \mathrm{m}^{-2}$ is required for P2 in order to explain the observed linear polarization at $43 \mathrm{GHz}$ (dashed red line). An even smaller $\sigma$ would be required if the intrinsic degree of polarization $m_{0}$ were smaller than $10 \%$ for a more disordered magnetic field. A $\sigma$ which is two orders of magnitude smaller than the RM (i.e., $\left.\epsilon \lesssim\left(2.5 \times 10^{4}\right) /\left(9 \times 10^{5}\right) \sim 0.03\right)$ indicates a highly uniform RM distribution in the external screen, which requires a well ordered large-scale magnetic field. Similar results are obtained for P1
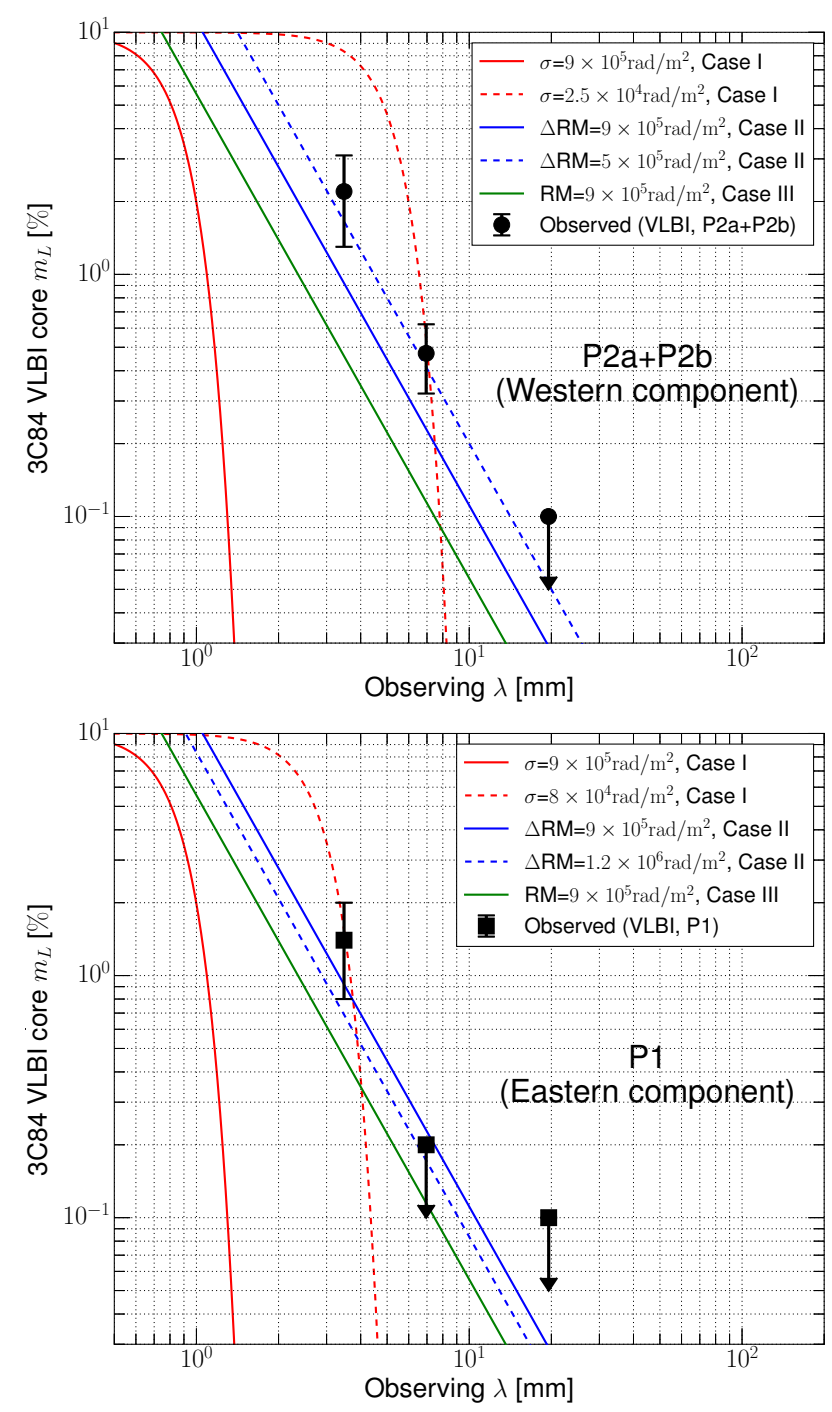

Fig. 13. Upper limits for $m_{\mathrm{L}}$ for external (Case I and II) and internal (Case III) Faraday depolarization and the VLBI polarization from observations. Arrows indicate the upper limits from observations. The top and bottom panels are for the P2 and P1 components, respectively. See the text for details.

$\left(\sigma\right.$ is at most $\sim 8 \times 10^{4} \mathrm{rad} \mathrm{m}^{-2}$ and $\left.\epsilon \lesssim\left(8 \times 10^{4}\right) /\left(9 \times 10^{5}\right) \sim 0.09\right)$. In contrast, Case II (blue lines) seems to explain the observed linear polarization in the core much better. We find that for P2 and $\mathrm{P} 1$ rotation measure gradients of $\Delta \mathrm{RM} \sim 5 \times 10^{5} \mathrm{rad} \mathrm{m}^{2}$ and $\Delta \mathrm{RM} \sim 1.2 \times 10^{6} \mathrm{rad} \mathrm{m}^{2}$ could explain the polarization at $\geq 43 \mathrm{GHz}$, respectively (dashed blue lines).

In Case III (green solid lines), the depolarization exceeds the values that we infer from our data and therefore we regard Case III as less likely and do not discuss it further.

Therefore, a smooth RM distribution in the external screen appears to be the most plausible scenario. We now proceed to explore the implications of the model cases I and II and try to distinguish between them.

\subsubsection{Case I : Accretion flow as the foreground screen}

In the vicinity of the central black hole and depending on its covering factor, a radiatively inefficient accretion flow (RIAF; e.g., Yuan \& Narayan 2014) could be regarded as a good candidate for a Faraday screen. In such a flow, magnetorotational instabilities and magnetohydrodynamic (MHD) 
turbulence can introduce a certain degree of inhomogenity in the matter and magnetic field distribution (Balbus \& Hawley 1991, 1998). Recently, Johnson et al. (2015) measured the linear polarization in Sgr A* with VLBI at $230 \mathrm{GHz}$ and suggested a short coherence length of the magnetic field orientation, of the order of $\sim 6 R_{\mathrm{s}}$. The linear polarization appears more random over larger scales. Adopting this also for the accretion flow of $3 \mathrm{C} 84$ leads to the expectation that the Faraday depth across the screen is comparable to the characteristic Faraday depth (i.e., $\sigma$ or $\Delta \mathrm{RM} \sim \mathrm{RM})$. However, this is in contrast to our finding (see Fig. 13). Certainly, our modeling simplifies a number of details in the disk-jet system. However, detailed general relativistic MHD simulations of such an accretion flow also suggest very strong depolarization of the jet by the accretion flow (e.g., Mościbrodzka et al. 2017). Hence, the accretion flow appears to us as a less preferred candidate for the Faraday screen.

Another problem in the association of the large RM in $3 \mathrm{C} 84$ with the accretion flow was pointed out by Plambeck et al. (2014). The authors modeled a spherical accretion flow in 3C 84 using a highly ordered, radial magnetic field, in order to relate the observed $\mathrm{RM} \sim 9 \times 10^{5} \mathrm{rad} \mathrm{m}^{-2}$ with the expected mass accretion rate. For realistic estimates of the magnetic field strength and the electron density in the accretion flow, the authors derive a higher RM, so that the observed RM appears to be too small. Therefore, the authors concluded that either (i) the magnetic field strength is much weaker than the equipartition value, (ii) the magnetic field is highly disordered, or (iii) the accretion flow is rather disk-like and the line of sight does not pass through the accretion flow. Indeed, an oblate disk-like geometry of the accretion flow would provide a suitable explanation because it explains the observational findings without fine-tuning the intrinsic physical properties of the accretion flow.

If the observed linear polarization is marginally affected by the disk-like accretion flow because of the geometry, the disk height $H$ to disk radius $R$ ratio $H / R$ should be $\tan ^{-1}(H / R) \lesssim$ $\left(90^{\circ}-\theta\right)$ or $H / R \lesssim \tan \left(90^{\circ}-\theta\right)$ where $\theta$ is the jet viewing angle. If we adopt $\theta \sim 30^{\circ}\left(\sim 60^{\circ}\right)$ from previous observations (Walker et al. 1994; Fujita \& Nagai 2017), we find $H / R \lesssim 1.7$ $(\lesssim 0.6)$. If the thick accretion flow is threaded by a strong poloidal magnetic field which can compress the disk vertically (e.g., McKinney et al. 2012), an even smaller $H / R$ ratio may be physically possible.

\subsubsection{Case II : Faraday rotation and depolarization due to the transverse jet stratification}

Alternatively, a mildly relativistic sheath surrounding the relativistic jet may provide the required external screen. A wide and collimated jet can be formed by the inner accretion disk or directly by a spinning central black hole (Blandford \& Payne 1982; Blandford \& Znajek 1977). The rotation of the central engine leads to the development of a stratified and twisted magnetic field topology (e.g., Tchekhovskoy et al. 2015). The boundary layer (or sheath) of such jets may provide the uniform external Faraday screen in Case II. Evidence for an ordered magnetic field configuration in jets comes from a plethora of polarization VLBI jet observations and from observations of transverse RM gradients on parsec scales (e.g., Zavala \& Taylor 2005; Hovatta et al. 2012; Gabuzda et al. 2017). The magnetic field is expected to be more ordered in the inner jet region when the jet-launching region becomes magnetically dominated (e.g., Zamaninasab et al. 2014; Martí-Vidal et al. 2015).

High-angular-resolution VLBI observations of the inner jet of 3C 84 show a limb-brightened morphology (see Fig. 7 and

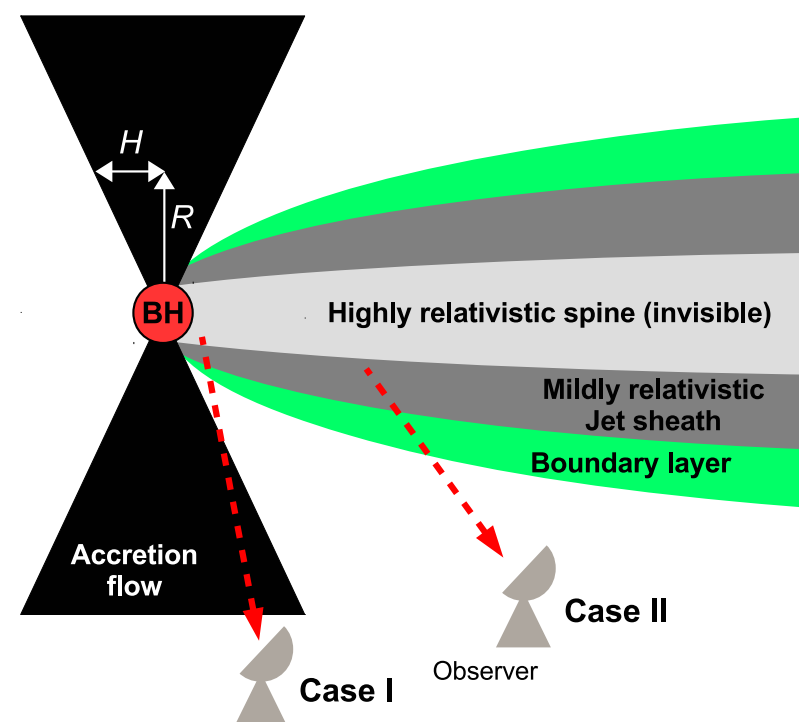

Fig. 14. Illustration of the geometry discussed throughout Sects. 4.3.1 and 4.3.2. We note that the figure is not drawn to scale.

also Giovannini et al. 2018). A limb-brightened jet most likely consists of at least two different jet components with different speeds, electron densities, or magnetic field strengths (spinesheath geometry; e.g., Pelletier \& Roland 1989; Komissarov 1990). Mildly relativistic electrons in the boundary layer of the jet, possibly mixed with thermal particles, will then cause Faraday rotation and conversion (Sokoloff et al. 1998; Perlman et al. 1999; Pushkarev et al. 2005; Porth et al. 2011; MacDonald et al. 2015; Pasetto et al. 2016; Lico et al. 2017). In Fig. 14 we show a sketch of a stratified jet for illustration.

\subsection{Estimation of the jet magnetic field strength and the electron density}

In the following we assume that the boundary layer of the jet is the Faraday screen and is responsible for the observed RM. We estimate the jet electron number density adopting the jet magnetic field strength from the synchrotron self-absorption theory. In convenient units, the observed RM can be written as follows:

$\frac{\mathrm{RM}}{\mathrm{rad} \mathrm{m}^{-2}}=8.1 \times 10^{5} \int_{\text {source }}^{\text {telescope }}\left(\frac{n_{\mathrm{e}}}{\mathrm{cm}^{-3}}\right)\left(\frac{B_{\|}}{G}\right)\left(\frac{\mathrm{d} l}{\mathrm{pc}}\right)$,

where $n_{\mathrm{e}}$ is the number density of the thermal electrons between the source and the telescope, $B_{\|}$is the line-of-sight component of the magnetic field and $\mathrm{d} l$ is the path length through the plasma from the source toward the observer.

We calculate the synchrotron self-absorption (SSA) magnetic field strength $B_{\mathrm{SSA}}$ in the core following Marscher (1983);

$B_{\mathrm{SSA}}=10^{-5} b(\alpha) \frac{\theta_{\mathrm{m}}^{4} v_{\mathrm{m}}^{5} \delta}{S_{\mathrm{m}}^{2}(1+z)}$ Gauss,

where $b(\alpha)$ is a constant tabulated in Marscher (1983) as a function of the spectral index, $S_{\mathrm{m}}$ is the flux density (in Jy) at the turnover frequency $v_{\mathrm{m}}$ (in $\mathrm{GHz}$ ), $\theta_{\mathrm{m}}$ is the source angular size (in mas) at $v_{\mathrm{m}}$ and $\delta$ is the Doppler factor. We note that the exact value of $v_{\mathrm{m}}$ is not accurately determined by our data (see the spectrum in Fig. 5). However, this spectrum and recent VLBI observations of $3 \mathrm{C} 84$ at $86 \mathrm{GHz}$ and $129 \mathrm{GHz}$ 
(Hodgson et al. 2018) suggest a spectral turnover near $86 \mathrm{GHz}$ Therefore, we adopt $v_{\mathrm{m}}=86 \mathrm{GHz}$ for the calculation of $B_{\mathrm{SSA}}$. For the optically thin spectrum we use $\alpha=-0.5$, which fixes $b(\alpha)=3.2$. Assuming $v_{\mathrm{m}}=86 \mathrm{GHz}, S_{\mathrm{m}}=(5.6 \pm 1.7) \mathrm{Jy}$ and $\theta_{\mathrm{m}}=1.8 \times(0.14 \pm 0.01) \mathrm{mas}^{7}$, we obtain $B_{\mathrm{SSA}} \sim(19 \pm 13) \times \delta \mathrm{G}$.

For the jet viewing angle $\theta \sim 30^{\circ}-60^{\circ}$ and the apparent jet speed $\sim 0.1 c$ near the core, the Doppler factor is $\delta \sim 1.1$. With this, we obtain $B_{\text {SSA }} \sim(21 \pm 14) \mathrm{G}$. We note that Abdo et al. (2009) and Aleksić et al. (2014) suggest a relatively large Doppler factor of $\delta \sim 2-4$ based on the theoretical modeling of the synchrotron self-Compton process in the jet. In this case, the resulting magnetic field strength would be $\sim(2-4)$ times larger.

We take $B_{\mathrm{SSA}}$ as the average magnetic field strength in the jet and calculate the average electron number density $n_{\mathrm{e}}$ by

$$
\frac{n_{\mathrm{e}}}{\mathrm{cm}^{-3}} \sim 1.2 \times 10^{-6}\left(\frac{\mathrm{RM}}{\mathrm{rad} \mathrm{m}^{-2}}\right)\left(\frac{B_{\mathrm{SSA}}}{G}\right)^{-1}\left(\frac{\theta_{\mathrm{m}}}{\mathrm{pc}}\right)^{-1},
$$

where we assume that the size $\theta_{\mathrm{m}}$, is a lower limit to the approximate path length for the jet medium because a longer path length is expected for a deprojected line-of-sight. For RM = $9 \times 10^{5} \mathrm{rad} \mathrm{m}^{-2}$, we obtain $n_{\mathrm{e}} \leq(0.6 \pm 0.4) \mathrm{cm}^{-3}$. The density will be even lower if the Doppler factor is larger and the magnetic field strength is higher. In any case, the density is an order of magnitude lower than an ambient gas density estimate of $n_{\mathrm{e}} \sim 8 \mathrm{~cm}^{-3}$ obtained by Fujita \& Nagai (2017) for the central parsecs. A low jet density is not implausible and is consistent with jet collimation by a denser ambient medium (e.g., Nagai et al. 2014; Giovannini et al. 2018).

We note that our calculations make simplified assumptions with regards to the magnetic field strength, the path length and the jet geometry. Future more detailed calculations using numerical radiative transfer across all Stokes parameters (e.g., MacDonald \& Marscher 2018) should be able to constrain these parameters more precisely. Also, the exact value of the turnover frequency on this spatial scale is still relatively uncertain. Quasisimultaneous global mm-VLBI observations with the GMVA and the Event Horizon Telescope (e.g., Doeleman et al. 2012; Lu et al. 2018) will help to determine the spectral properties of the innermost jet and central engine.

\section{Summary and conclusions}

In this paper, we presented a study of the polarization properties of the radio galaxy $3 \mathrm{C} 84$ based on the results from polarimetric VLBI observations of the source at mm-wavelengths. We summarize our findings and main conclusions as follows:

1. We found asymmetrically distributed and polarized emission in the VLBI core region of $3 \mathrm{C} 84$ at $86 \mathrm{GHz}$ using GMVA observations. The east-west oriented and linearly polarized structure consists of two polarized components (P1 and $\mathrm{P} 2$ ), which are separated by $\sim 0.2$ mas (corresponding to $\sim 0.07 \mathrm{pc} \sim 812 R_{\mathrm{S}}$ ). The fractional linear polarization $m_{\mathrm{L}}$ of $\mathrm{P} 1$ and $\mathrm{P} 2$ at $86 \mathrm{GHz}$ is $\sim 2 \%$ with the VLBA $43 \mathrm{GHz}$ beam.

2. Additional close-in-time VLBA $43 \mathrm{GHz}$ polarization images also reveal the presence of polarized emission $\left(m_{\mathrm{L}} \sim\right.$ $0.4-0.5 \%)$. The polarized structure is also time-variable. The moving direction of the variable polarized emission feature, which is almost perpendicular to the milliarcsecond jet, suggests that the jet base may be edge-brightened in polarized light. However, other effects such as ejection of a new

The factor 1.8 corrects for the geometry (Marscher 1983).
VLBI component or opacity effects across the jet cannot be excluded.

3. The total intensity spectrum of $3 \mathrm{C} 84$ is inverted up to at least $86 \mathrm{GHz}$ and the linear polarization $m_{\mathrm{L}}$ increases with frequency following a power-law $m_{\mathrm{L}} \propto v^{0.77}$. A likely explanation invokes a decreasing synchrotron opacity and lower Faraday depolarization at higher frequencies.

4. The combination of quasi-simultaneous EVPA measurements at 43 and $86 \mathrm{GHz}$ and adding near in time EVPA measurements from ALMA at higher frequencies, reveals a high rotation measure in the VLBI core region of RM $\sim 2 \times 10^{5} \mathrm{rad} \mathrm{m}^{-2}$ at $\gtrsim 43 \mathrm{GHz}$ in May 2015 . This is comparable to the RM measurements at $230 \mathrm{GHz}$ on larger angular scales (Plambeck et al. 2014).

5. A significant rotation of the EVPA is also observed within the $43 \mathrm{GHz}$ VLBA observing band and also suggests the presence of Faraday rotation. Repeated measurements of the $\mathrm{RM}$ within the $43 \mathrm{GHz}$ VLBA observing band are consistent with the high RM in the VLBI core region at three epochs, but also suggest that the RM has changed its sign at one epoch. This could be related to the onset of a small total intensity flare in the VLBI core in 2015.

6. In order to explain the degree of linear polarization by Faraday depolarization, an external screen with either a small RM dispersion or only a smoothly varying uniform RM distribution is required.

7. The data would support a tentative association of the Faraday screen with the accretion flow if (i) the accretion flow was found to be thick $(H / R \geq 1.7$ for the jet viewing angle $\theta=30^{\circ}$ ) and more importantly (ii) the magnetic field was highly ordered. However, the latter appears to be in contradiction with the results of previous studies (e.g., Plambeck et al. 2014). Instead, a stratified jet with a boundary layer containing an ordered magnetic field configuration could also provide a good explanation for the large RM, the frequency dependence of the depolarization and possibly also for the RM variability. The edge-brightened jet morphology observed at $22 \mathrm{GHz}$ (Giovannini et al. 2018) would also support this interpretation.

8. From synchrotron self-absorption theory, we calculate a magnetic field strength in the VLBI core region of 3C 84 of $B_{\text {SSA }}=(21 \pm 14) \mathrm{G}$, adopting an only mild relativistic beaming with a Doppler factor of $\delta \sim 1.1$ from jet kinematics. In this case a jet electron density of $n_{\mathrm{e}} \leq(0.6 \pm 0.4) \mathrm{cm}^{-3}$ is required to explain the observed high $\mathrm{RM}$. The field strength would be larger by factor $\sim 2-4$ if a higher Doppler factor of $\delta \sim 2-4$ is used and the electron density will be correspondingly lower. In both cases the jet electron density appears to be at least an order of magnitude lower than the density of the ambient gas.

Overall, our study suggests that either the accretion flow or a transversely stratified jet with boundary layers can play an important role in the generation of the polarized mm-wave emission in the milliparsec scale VLBI structure. Future millimeter VLBI imaging, performed quasi-simultaneously at different frequencies and including VLBI observations above $86 \mathrm{GHz}$, will help to better understand the complicated nature of the polarization in 3C 84. Also, a more detailed theoretical modeling of the linear and circular polarization spectrum and complemented by numerical simulations, would be very beneficial. Finally, we note that the detection of significant linear polarization at the jet base in 3C 84 may shed light on the properties of the Faraday screen in other radio galaxies (e.g., M 87; Kim et al. 2018). Further studies should prove fruitful. 
Acknowledgements. We thank the anonymous referee for valuable comment and suggestions, which greatly helped to improve the paper. We thank Carolina Casadio and Ioannis Myserlis for fruitful discussions and comments. J.-Y.K. is supported for this research by the International Max-Planck Research School (IMPRS) for Astronomy and Astrophysics at the University of Bonn and Cologne. I.A. acknowledges support by a Ramón y Cajal grant of the Ministerio de Economía, Industria y Competitividad (MINECO) of Spain and by an additional MINECO grant with reference AYA2016-80889-P. R.-S. L. is supported by the National Youth Thousand Talents Program of China and by the Max-Planck Partner Group. This research has made use of data obtained with the Global Millimeter VLBI Array (GMVA), which consists of telescopes operated by the MPIfR, IRAM, Onsala, Metsahovi, Yebes, the Korean VLBI Network, the Green Bank Observatory and the Long Baseline Observatory. The VLBA is an instrument of the Long Baseline Observatory, which is a facility of the National Science Foundation operated by Associated Universities, Inc. The data were correlated at the correlator of the MPIfR in Bonn, Germany. This work made use of the Swinburne University of Technology software correlator, developed as part of the Australian Major National Research Facilities Programme and operated under licence. This study makes use of 43 GHz VLBA data from the VLBA-BU Blazar Monitoring Program (VLBA-BUBLAZAR; http: //www.bu.edu/blazars/VLBAproject.html), funded by NASA through the Fermi Guest Investigator Program. This research has made use of data from the MOJAVE database that is maintained by the MOJAVE team (Lister et al. 2009). This paper makes use of the following ALMA data: ADS/JAO.ALMA\#2011.0.00001.CAL. ALMA is a partnership of ESO (representing its member states), NSF (USA) and NINS (Japan), together with NRC (Canada), MOST and ASIAA (Taiwan) and KASI (Republic of Korea), in cooperation with the Republic of Chile. The Joint ALMA Observatory is operated by ESO, AUI/NRAO and NAOJ. This research has made use of data from the OVRO $40 \mathrm{~m}$ monitoring program (Richards et al. 2011). The OVRO $40 \mathrm{M}$ Telescope Fermi Blazar Monitoring Program is supported by NASA under awards NNX08AW31G and NNX11A043G, and by the NSF under awards AST0808050 and AST-1109911.

\section{References}

Abdo, A. A., Ackermann, M., Ajello, M., et al. 2009, ApJ, 699, 31 Agudo, I., Thum, C., Wiesemeyer, H., \& Krichbaum, T. P. 2010, ApJS, 189, 1 Agudo, I., Marscher, A. P., Jorstad, S. G., et al. 2012, ApJ, 747, 63

Agudo, I., Thum, C., Gómez, J. L., \& Wiesemeyer, H. 2014, A\&A, 566, A59 Agudo, I., Thum, C., Molina, S. N., et al. 2018a, MNRAS, 474, 1427 Agudo, I., Thum, C., Ramakrishnan, V., et al. 2018b, MNRAS, 473, 1850 Aleksić, J., Ansoldi, S., Antonelli, L. A., et al. 2014, A\&A, 564, A5 Aller, H. D., Aller, M. F., \& Plotkin, R. M. 2003, Ap\&SS, 288, 17

Balbus, S. A., \& Hawley, J. F. 1991, ApJ, 376, 214

Balbus, S. A., \& Hawley, J. F. 1998, Rev. Mod. Phys., 70, 1

Blandford, R. D., \& Payne, D. G. 1982, MNRAS, 199, 883

Blandford, R. D., \& Znajek, R. L. 1977, MNRAS, 179, 433

Boccardi, B., Krichbaum, T. P., Ros, E., \& Zensus, J. A. 2017, A\&ARv, 25, 4

Bower, G. C., Falcke, H., Sault, R. J., \& Backer, D. C. 2002, ApJ, 571, 843

Burn, B. J. 1966, MNRAS, 133, 67

Casadio, C., Gómez, J. L., Jorstad, S. G., et al. 2015, ApJ, 813, 51

Casadio, C., Krichbaum, T., Marscher, A., et al. 2017, Galaxies, 5, 67

Clausen-Brown, E., Lyutikov, M., \& Kharb, P. 2011, MNRAS, 415, 2081

Deller, A. T., Brisken, W. F., Phillips, C. J., et al. 2011, Publ. Astron. Soc. Pac., 123,275

Doeleman, S. S., Fish, V. L., Schenck, D. E., et al. 2012, Science, 338, 355

Fujita, Y., \& Nagai, H. 2017, MNRAS, 465, L94

Gabuzda, D. C., Roche, N., Kirwan, A., et al. 2017, MNRAS, 472, 1792

Giovannini, G., Savolainen, T., Orienti, M., et al. 2018, Nat. Astron., 33

Gómez, J. L., Marscher, A. P., Jorstad, S. G., Agudo, I., \& Roca-Sogorb, M 2008, ApJ, 681, L69

Gómez, J. L., Lobanov, A. P., Bruni, G., et al. 2016, ApJ, 817, 96

Greisen, E. W. 1990, in Acquisition, Processing and Archiving of Astronomical, eds. G. Longo, \& G. Sedmak, 125

Hodgson, J. A., Krichbaum, T. P., Marscher, A. P., et al. 2017, A\&A, 597, A80

Hodgson, J. A., Rani, B., Lee, S.-S., et al. 2018, MNRAS, 475, 368

Homan, D. C., \& Wardle, J. F. C. 2004, ApJ, 602, L13

Hovatta, T., Lister, M. L., Aller, M. F., et al. 2012, AJ, 144, 105

Jackson, N., Browne, I. W. A., Battye, R. A., Gabuzda, D., \& Taylor, A. C. 2010 , MNRAS, 401, 1388

Johnson, M. D., Fish, V. L., Doeleman, S. S., et al. 2015, Science, 350, 1242
Jones, T. W., \& Odell, S. L. 1977, ApJ, 214, 522

Jorstad, S. G., Marscher, A. P., Lister, M. L., et al. 2005, AJ, 130, 1418 Jorstad, S. G., Marscher, A. P., Stevens, J. A., et al. 2007, AJ, 134, 799 Jorstad, S. G., Marscher, A. P., Morozova, D. A., et al. 2017, ApJ, 846, 98

Kim, J. Y., Krichbaum, T. P., Lu, R. S., et al. 2018, A\&A, 616, A188 Komissarov, S. S. 1990, Sov. Astron. Lett., 16, 284

Koyama, S., Kino, M., Giroletti, M., et al. 2016, A\&A, 586, A113

Leppanen, K. J., Zensus, J. A., \& Diamond, P. J. 1995, AJ, 110, 2479

Lico, R., Gómez, J. L., Asada, K., \& Fuentes, A. 2017, MNRAS, 469, 1612

Lister, M. L., \& Homan, D. C. 2005, AJ, 130, 1389

Lister, M. L., Aller, H. D., Aller, M. F., et al. 2009, AJ, 137, 3718

Lobanov, A. P. 1998, A\&A, 330, 79

Lu, R.-S., Krichbaum, T. P., Roy, A. L., et al. 2018, ApJ, 859, 60

Lyutikov, M., Pariev, V. I., \& Gabuzda, D. C. 2005, MNRAS, 360, 869

MacDonald, N. R., \& Marscher, A. P. 2018, ApJ, 862, 58

MacDonald, N. R., Marscher, A. P., Jorstad, S. G., \& Joshi, M. 2015, ApJ, 804, 111

Marscher, A. P. 1983, ApJ, 264, 296

Marscher, A. P. 2014, ApJ, 780, 87

Martí-Vidal, I., Krichbaum, T. P., Marscher, A., et al. 2012, A\&A, 542, A107

Martí-Vidal, I., Muller, S., Vlemmings, W., Horellou, C., \& Aalto, S. 2015, Science, 348,311

McKinney, J. C., Tchekhovskoy, A., \& Blandford, R. D. 2012, MNRAS, 423, 3083

Montier, L., Plaszczynski, S., Levrier, F., et al. 2015a, A\&A, 574, A135

Montier, L., Plaszczynski, S., Levrier, F., et al. 2015b, A\&A, 574, A136

Mościbrodzka, M., Dexter, J., Davelaar, J., \& Falcke, H. 2017, MNRAS, 468, 2214

Muñoz, D. J., Marrone, D. P., Moran, J. M., \& Rao, R. 2012, ApJ, 745, 115

Myserlis, I., Angelakis, E., Kraus, A., et al. 2018, A\&A, 609, A68

Nagai, H., Haga, T., Giovannini, G., et al. 2014, ApJ, 785, 53

Nagai, H., Fujita, Y., Nakamura, M., et al. 2017, ApJ, 849, 52

O'Sullivan, S. P., \& Gabuzda, D. C. 2009, MNRAS, 393, 429

Pacholczyk, A. G. 1970, Radio Astrophysics. Nonthermal Processes in Galactic and Extragalactic Sources (San Francisco: Freeman)

Pasetto, A., Carrasco-González, C., Bruni, G., et al. 2016, Galaxies, 4, 66

Pelletier, G., \& Roland, J. 1989, A\&A, 224, 24

Perlman, E. S., Biretta, J. A., Zhou, F., Sparks, W. B., \& Macchetto, F. D. 1999, AJ, 117, 2185

Plambeck, R. L., Bower, G. C., Rao, R., et al. 2014, ApJ, 797, 66

Porth, O., Fendt, C., Meliani, Z., \& Vaidya, B. 2011, ApJ, 737, 42

Pushkarev, A. B., Gabuzda, D. C., Vetukhnovskaya, Y. N., \& Yakimov, V. E. 2005, MNRAS, 356, 859

Rani, B., Krichbaum, T. P., Marscher, A. P., et al. 2015, A\&A, 578, A123

Richards, J. L., Max-Moerbeck, W., Pavlidou, V., et al. 2011, ApJS, 194, 29

Roberts, D. H., Wardle, J. F. C., \& Brown, L. F. 1994, ApJ, 427, 718

Scharwächter, J., McGregor, P. J., Dopita, M. A., \& Beck, T. L. 2013, MNRAS, 429, 2315

Schinzel, F. K., Lobanov, A. P., Taylor, G. B., et al. 2012, A\&A, 537, A70

Shepherd, M. C., Pearson, T. J., \& Taylor, G. B. 1994, Bull. Am. Astron. Soc., 26, 987

Sokoloff, D. D., Bykov, A. A., Shukurov, A., et al. 1998, MNRAS, 299, 189

Strauss, M. A., Huchra, J. P., Davis, M., et al. 1992, ApJS, 83, 29

Suzuki, K., Nagai, H., Kino, M., et al. 2012, ApJ, 746, 140

Taylor, G. B., Gugliucci, N. E., Fabian, A. C., et al. 2006, MNRAS, 368, 1500

Tchekhovskoy, A. 2015, in The Formation and Disruption of Black Hole Jets, eds. I. Contopoulos, D. Gabuzda, \& N. Kylafis, Astrophysics and Space Science Library, 414, 45

Thompson, A. R., Moran, J. M., \& Swenson, G. W. 2017, Interferometry and Synthesis in Radio Astronomy, 3rd edn.

Thum, C., Wiesemeyer, H., Paubert, G., Navarro, S., \& Morris, D. 2008, PASP, 120,777

Thum, C., Agudo, I., Molina, S. N., et al. 2018, MNRAS, 473, 2506

Trippe, S., Bremer, M., Krichbaum, T. P., et al. 2012, MNRAS, 425, 1192

Walker, R. C., Romney, J. D., \& Benson, J. M. 1994, ApJ, 430, L45

Walker, R. C., Dhawan, V., Romney, J. D., Kellermann, K. I., \& Vermeulen, R. C. 2000, ApJ, 530, 233

Wardle, J. F. C., \& Homan, D. C. 2003, Ap\&SS, 288, 143

Wardle, J. F. C., \& Kronberg, P. P. 1974, ApJ, 194, 249

Yuan, F., \& Narayan, R. 2014, ARA\&A, 52, 529

Zamaninasab, M., Clausen-Brown, E., Savolainen, T., \& Tchekhovskoy, A. 2014, Nature, 510, 126

Zavala, R. T., \& Taylor, G. B. 2005, ApJ, 626, L73 


\section{Appendix A: Flux density calibration of 3 C 84 using $86 \mathrm{GHz}$ GMVA data in May 2015}

Here we discuss a method to improve the accuracy of the absolute flux density calibration of $3 \mathrm{C} 84$ at $86 \mathrm{GHz}$ based on the available a-priori GMVA amplitude calibration, which is not accurate enough (see also Koyama et al. 2016). We compared the multifrequency VLBI flux densities of 3C 84 (Sects. 2.1 and 2.2) with the total flux densities measured by other instruments. At $v=15 \mathrm{GHz}$, the Owens Valley Radio Observatory (OVRO; Richards et al. 2011) monitoring program ${ }^{8}$ provided near-in-time flux density measurements of $S=31.8 \pm 0.5 \mathrm{Jy}$ and $S=32.3 \pm 0.1 \mathrm{Jy}$ for 3C 84 on 03 Apr 2015 and 27 Jun 2015, respectively. We averaged the two fluxes in order to estimate the total flux in May 2015 and took half of their difference as the associated error, finding $S=32.1 \pm 0.3 \mathrm{Jy}$. At $v \gtrsim 86 \mathrm{GHz}$, we refer to Sect. 2.4 for the ALMA flux density measurements. In Fig. A.1 we show (i) the VLBI-scale flux density of the source within $\sim 4$ mas from the core $\left(S_{\mathrm{VLBI}}\right)$, (ii) the VLBI-scale flux density only for the extended jet ( $\sim 2-4$ mas from the core; $\left.S_{\text {Jet }}\right)$ and (iii) the total (i.e., spatially unresolved) flux density measured by the OVRO and the ALMA observations $\left(S_{\text {tot }}\right)$. The GMVA flux densities were taken from only the self-calibrated data, based on the a-priori calibration. We fitted a power-law model to the total flux density (i.e., $S_{\mathrm{VLBI}} \propto v^{+\alpha}$ ) in order to estimate the VLBI flux density $S_{\text {VLBI }}$ at 43 and $86 \mathrm{GHz}$. The total flux density spectrum is fitted by $S_{\text {tot }} \propto v^{-(0.45 \pm 0.04)}$. From this we estimate $S_{\text {tot }}$ at 43 and $86 \mathrm{GHz}$ and calculated the VLBIto-total flux density ratio $S_{\mathrm{VLBI}} / S_{\text {tot }}$ (i.e., the compactness). At 15 and $43 \mathrm{GHz}$, the flux ratio is comparable $(\sim 0.90$ and $\sim 0.84$ at $15 \mathrm{GHz}$ and $43 \mathrm{GHz}$, respectively). However at $86 \mathrm{GHz}$, it is much lower $\left(S_{\mathrm{VLBI}} / S_{\text {tot }} \sim 0.40\right)$. The comparison of the spectral index of the extended jet emission yields a spectral index of $\alpha_{15-43 \mathrm{GHz}} \sim-0.5$, while the spectral index from 43 to $86 \mathrm{GHz}$ is much steeper; $\alpha_{43-86 \mathrm{GHz}} \sim-1.8$. Such a steep spectrum is unlikely and is inconsistent with ALMA measurements between 98 and $344 \mathrm{GHz}$. We therefore take this as another indication of overly low flux densities at $86 \mathrm{GHz}$.

To check for an overall scaling problem of the GMVA amplitude calibration we examined the compactness ratio $S_{\mathrm{VLBI}} / S_{\text {tot }}$ also for other calibrators at $86 \mathrm{GHz}$ (3C 454.3, BLLac, CTA 102 and OJ 287), for which we find - applying a similar procedure as described above - compactness ratios of typically 0.9 at 15 and $43 \mathrm{GHz}$, but $\sim 0.5$ at $86 \mathrm{GHz}$. In the following we assume

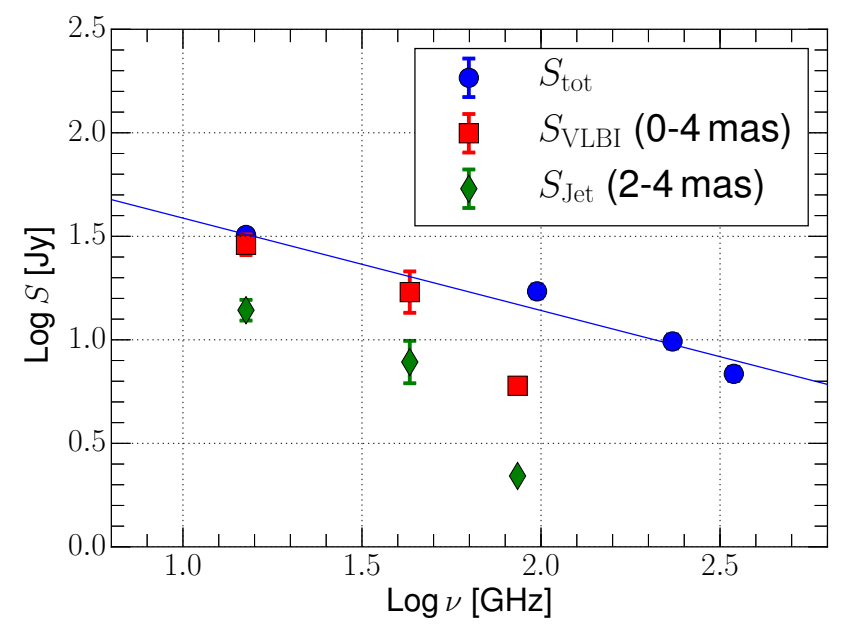

Fig. A.1. Quasi-simultaneous total and VLBI-scale flux densities of 3C 84 in May 2015 displayed in log-log scale. The blue solid line shows the power-law fitted to the total flux measurements. We omit the error bars for the GMVA $86 \mathrm{GHz}$ data points.

that the true compactness $S_{\mathrm{VLBI}} / S_{\text {tot }}$ at 15,43 and $86 \mathrm{GHz}$ is comparable. Then the visibility amplitudes of the GMVA data of 3C 84 can be upscaled by a factor $g \sim 2.1-2.3$. Confidence for this number also comes from similar factors obtained for the other calibrators. With this correction factor a much more realistic value of the (optically thin) jet spectral index $\alpha_{43-86 \mathrm{GHz}} \sim-0.8$ is obtained. The corrected total flux density $(\sim 12 \mathrm{Jy})$ is now also in good agreement with other near-in-time VLBI flux density measurements at $86 \mathrm{GHz}$ reported in literature (e.g., $S \sim(10-15)$ Jy; see Hodgson et al. 2018).

In the following we discuss the uncertainty $\Delta g$ of the scaling value $g$, which depends on the validity of our assumptions and we could alternatively assume the same spectral index from $15 \mathrm{GHz}$ to $86 \mathrm{GHz}$ for $S_{\text {Jet }}$. Assuming a constant spectral index $\alpha_{15-86 \mathrm{GHz}} \sim-0.5$ results in a slightly larger correction factor of $g \sim 2.5$. The range of the $g \sim 2.1-2.5$ suggests that its uncertainty is $\Delta g \sim 0.4$, corresponding to a $\sim 20 \%$ of error in the absolute flux density. It would be overly optimistic to claim an error smaller than $\sim 20 \%$ without having identified the origin of the amplitude scaling error. Therefore, we adopted a slightly larger value of $\sim 30 \%$ for the systematic uncertainty in the measured VLBI flux densities (for this particular epoch).

\footnotetext{
8 http://www.astro.caltech.edu/ovroblazars/
} 


\section{Appendix B: Robustness of the polarization imaging at $86 \mathrm{GHz}$}
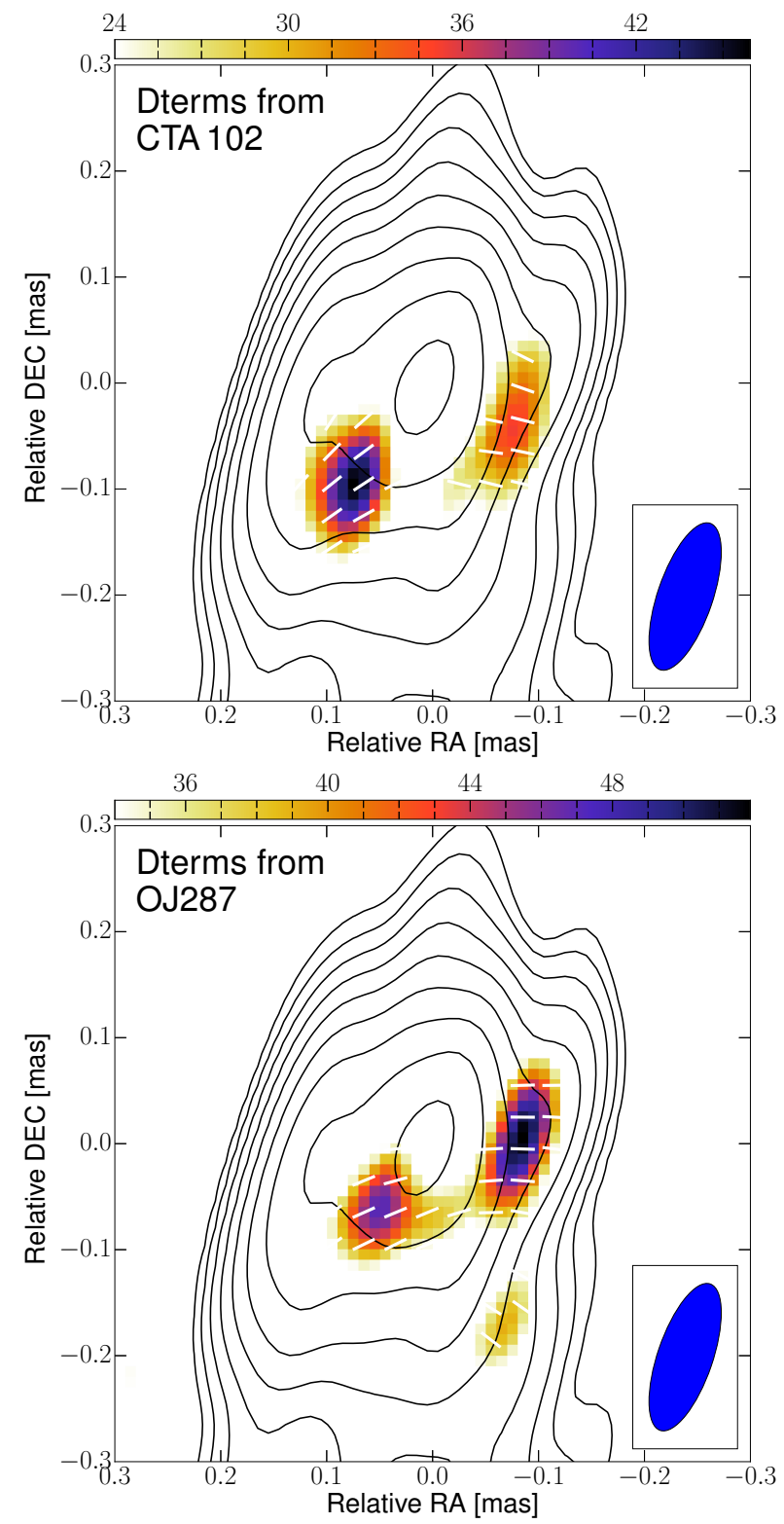

Fig. B.1. Linear polarization maps of the nuclear region of $3 \mathrm{C} 84$ at $86 \mathrm{GHz}$ obtained with different polarization calibrations. The two maps were reconstructed using the D-terms obtained from CTA 102 (upper panel) and from OJ 287 (lower panel), respectively. The minimum contour level is $7 \mathrm{mJy}^{\text {beam }}{ }^{-1}$.

In order to illustrate the impact of slightly different D-terms on the polarization image of the GMVA data, we made different linear polarization images of 3C 84 using D-terms determined from the individual calibrators. Two examples are shown in Fig. B.1. We find that two central polarization features are reproduced in the nuclear region at similar locations with similar EVPAs. We note that the structure of the jet of OJ 287 is substantially different from that of CTA 102 (e.g., Agudo et al. 2012; Casadio et al. 2015; Hodgson et al. 2017). Despite this, the two polarization maps shown in Fig. B.1 look similar, which provides confidence of the polarization calibration and imaging of 3C 84 . Nevertheless, we consider that the polarization image in the main text, which is obtained with the averaged D-terms, is of better quality than those in Fig. B.1 for the reasons explained in the main text.

\section{Appendix C: Multiepoch VLBA $43 \mathrm{GHz}$ data}

Table C.1. Properties of the polarized components at $43 \mathrm{GHz}$ obtained from the polarization imaging at four different IFs.

\begin{tabular}{|c|c|c|c|c|}
\hline $\begin{array}{c}\text { Epoch } \\
(1) \\
{[\text { yyyy/mm/dd] }}\end{array}$ & $\begin{array}{c}v_{\mathrm{obs}} \\
(2) \\
{[\mathrm{GHz}]} \\
\end{array}$ & $\begin{array}{l}S_{v}{ }^{a} \\
(3) \\
{[\mathrm{Jy}]} \\
\end{array}$ & $\begin{array}{l}m_{\mathrm{L}} \\
(4) \\
{[\%]} \\
\end{array}$ & $\begin{array}{c}\chi \\
(5) \\
{[\mathrm{deg}]}\end{array}$ \\
\hline \multirow[t]{4}{*}{$2015 / 04 / 11$} & 43.0075 & $1.4 \pm 0.1$ & $0.73 \pm 0.23$ & $3 \pm 10$ \\
\hline & 43.0875 & & $0.76 \pm 0.24$ & $2 \pm 10$ \\
\hline & 43.1515 & & $0.61 \pm 0.19$ & $8 \pm 10$ \\
\hline & 43.2155 & & $0.32 \pm 0.10$ & $20 \pm 10$ \\
\hline \multirow[t]{4}{*}{$2015 / 05 / 11$} & 43.0075 & $2.0 \pm 0.2$ & $0.44 \pm 0.14$ & $-(9 \pm 10)$ \\
\hline & 43.0875 & & $0.49 \pm 0.16$ & $-(20 \pm 10)$ \\
\hline & 43.1515 & & $0.53 \pm 0.17$ & $-(24 \pm 10)$ \\
\hline & 43.2155 & & $0.43 \pm 0.13$ & $-(15 \pm 10)$ \\
\hline \multirow[t]{4}{*}{$2015 / 07 / 02$} & 43.0075 & $3.6 \pm 0.4$ & $0.37 \pm 0.12$ & $-(5 \pm 10)$ \\
\hline & 43.0875 & & $0.40 \pm 0.13$ & $-(22 \pm 10)$ \\
\hline & 43.1515 & & $0.67 \pm 0.21$ & $-(26 \pm 10)$ \\
\hline & 43.2155 & & $0.47 \pm 0.15$ & $-(23 \pm 10)$ \\
\hline \multirow[t]{4}{*}{$2015 / 09 / 22$} & 43.0075 & $2.8 \pm 0.3$ & $0.28 \pm 0.09$ & $-(4 \pm 10)$ \\
\hline & 43.0875 & & $0.37 \pm 0.10$ & $6 \pm 10$ \\
\hline & 43.1515 & & $0.33 \pm 0.11$ & $-(10 \pm 10)$ \\
\hline & 43.2155 & & $0.38 \pm 0.12$ & $-(15 \pm 10)$ \\
\hline
\end{tabular}

Notes. The columns show (1) the observing epoch, (2) the observing frequency at each IF, (3) the total flux, (4) the degree of linear polarization, and (5) the EVPA. The total flux was consistent across the frequency in each epoch and we report the measurement only for a single IF. ${ }^{(a)}$ The time-variable total flux density of the polarized component is due to not only the flux variability but also the different positions of the peak of the polarization.

Table C.2. The unpolarized core flux (in Jy), the peak (in Jy beam ${ }^{-1}$ ) and the core FWHM size (in mas) during 2015 measured from the archival VLBA $43 \mathrm{GHz}$ data sets by the model-fitting analysis.

\begin{tabular}{cccc}
\hline \hline $\begin{array}{c}\text { Epoch } \\
\text { [yyyy/mm/dd] }\end{array}$ & $\begin{array}{c}\text { Flux } \\
{[\mathrm{Jy}]}\end{array}$ & $\begin{array}{c}\text { Peak } \\
{\left[\text { Jy beam }^{-1} \text { ] }\right.}\end{array}$ & $\begin{array}{c}\text { FWHM size } \\
{[\mathrm{mas}]}\end{array}$ \\
\hline $2015 / 02 / 14$ & $3.9 \pm 0.7$ & $3.6 \pm 0.5$ & $0.12 \pm 0.01$ \\
$2015 / 04 / 11$ & $4.5 \pm 0.6$ & $4.1 \pm 0.4$ & $0.13 \pm 0.01$ \\
$2015 / 05 / 11$ & $4.8 \pm 0.7$ & $4.4 \pm 0.4$ & $0.13 \pm 0.01$ \\
$2015 / 06 / 09$ & $5.8 \pm 0.6$ & $5.2 \pm 0.4$ & $0.14 \pm 0.01$ \\
$2015 / 07 / 02$ & $5.8 \pm 0.6$ & $5.2 \pm 0.4$ & $0.16 \pm 0.01$ \\
$2015 / 08 / 01$ & $5.0 \pm 0.5$ & $4.5 \pm 0.3$ & $0.16 \pm 0.01$ \\
$2015 / 09 / 22$ & $5.0 \pm 0.6$ & $4.4 \pm 0.4$ & $0.16 \pm 0.01$ \\
$2015 / 12 / 05$ & $4.1 \pm 0.5$ & $3.7 \pm 0.3$ & $0.15 \pm 0.01$ \\
\hline
\end{tabular}

Notes. A circular beam of 0.3 mas was used for all epochs.

In Table C. 1 we show the results of the VLBA $43 \mathrm{GHz}$ polarization measurements in the four different epochs. We provide the polarization maps of the corresponding epochs obtained from the frequency-averaged visibilities in Fig. C.1. The core flux and the FWHM size during 2015 are given in Table C.2. 

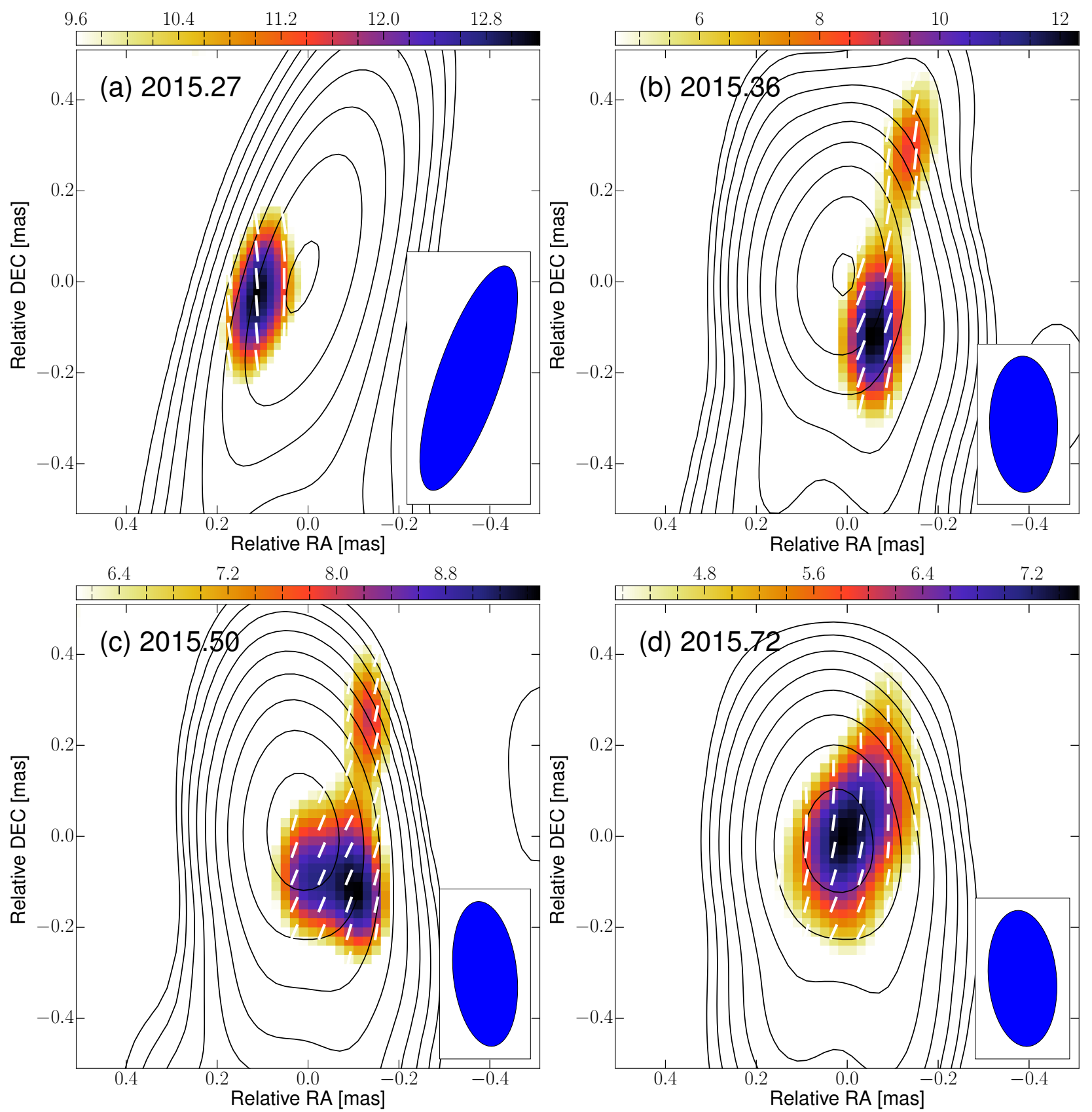

Fig. C.1. VLBA $43 \mathrm{GHz}$ polarization images of 3C 84 zoomed into the core. Labels in the upper left of each panel denotes the observing epoch. The contours show the total intensity and start from $25,7,10$ and $20 \mathrm{mJy}_{\text {beam }}{ }^{-1}$ and increase by factor of two for the panels $a, b, c$ and $d$, respectively. The color bar indicates the linear polarization intensity in mJy beam ${ }^{-1}$. 